Pacific Journal of Mathematics

KRILL DIMENSION OF SKEW-LAURENT EXTENSIONS 


\title{
KRULL DIMENSION OF SKEW-LAURENT EXTENSIONS
}

\author{
K. R. GoOdearl AND T. H. Lenagan
}

A precise formula is derived for the (noncommutative) Krull dimension of a skew-Laurent extension $R\left[\theta_{1}^{ \pm 1}, \ldots, \theta_{u}^{ \pm 1}\right]$, where $R$ is a commutative noetherian ring of finite Krull dimension, equipped with $u$ commuting automorphisms $\sigma_{1}, \ldots, \sigma_{u}$. The formula is given in terms of heights and automorphian dimensions of prime ideals of $R$, where the automorphian dimension of a prime ideal $P$ is a positive integer that measures the invariance of $P$ relative to products of powers of the $\sigma_{i}$. As part of the development of this formula, the Krull dimension of a skew-Laurent extension $R\left[\theta_{1}^{ \pm 1}\right]$ over a right noetherian ring $R$ of finite right Krull dimension is determined. Also, some partial results are obtained for an iterated skew-Laurent extension $R\left[\theta_{1}^{ \pm 1}, \ldots, \theta_{u}^{ \pm 1}\right]$ over a right noetherian ring $R$ of finite right Krull dimension. In particular, a criterion is derived that indicates when such an iterated skew-Laurent extension can achieve the maximum possible Krull dimension.

Introduction. This paper is concerned with the Krull dimension (in the sense of Rentschler and Gabriel) of a skew-Laurent extension $T=$ $R\left[\theta_{1}^{ \pm 1}, \ldots, \theta_{u}^{ \pm 1}\right]$, where $R$ is a commutative noetherian ring of finite Krull dimension, equipped with $u$ commuting automorphisms $\sigma_{1}, \ldots, \sigma_{u}$. The main theorem states that the Krull dimension of $T$ equals the maximum of the values

$$
\text { height }(P)+\text { aut.dim. }(P)
$$

as $P$ ranges over the prime ideals of $R$, where aut.dim. $(P)$ is a nonnegative integer that measures the invariance of $P$ relative to products of powers of the $\sigma_{l}$. This theorem is developed in Part $\mathrm{C}$ of the paper. Part A is concerned with the question of the Krull dimension of a skew-Laurent extension $T=R\left[\theta, \theta^{-1}\right]$ over a right noetherian ring of finite right Krull dimension $n$, equipped with a single automorphism $\sigma$. In this portion of the paper, the main result is that $T$ has Krull dimension $n$ unless there exists a simple right $R$-module $A$ such that $A \otimes_{R} T$ is not simple (as a $T$-module) and $A$ has height $n$ in the sense that there exist critical right $R$-modules $A=A_{0}, A_{1}, \ldots, A_{n}$ such that each $A_{i} \otimes_{R} T$ is a critical $T$-module, each $A_{i}$ is a minor subfactor of $A_{i+1}$, and $A_{n}$ is a subfactor of $R$. If such an $A$ does exist, then $T$ has Krull dimension $n+1$. This criterion is simplified when $R$ is fully bounded. In this case it is shown that $T$ has Krull dimension $n$ unless $R$ has a maximal two-sided ideal $M$ of height $n$ 
such that $M$ is invariant under some nonzero power of $\sigma$, in which case $T$ has Krull dimension $n+1$. In Part B of the paper, some technical results are developed that relate to the Krull dimension of an iterated skewLaurent extension $T=R\left[\theta_{1}^{ \pm 1}, \ldots, \theta_{u}^{ \pm 1}\right]$ over a right noetherian ring $R$ with finite right Krull dimension $k$. Although the main thrust of these results is toward applications in Part C, they do lead to a criterion that tells when $T$ can have the maximum possible Krull dimension, namely $k+u$.

\section{A. Noncommutative Coefficients; Single Automorphism}

If $R$ is a right noetherian ring and $\sigma$ is an automorphism of $R$, then we can construct the skew-Laurent extension $T=R\left[\theta, \theta^{-1} ; \sigma\right]$, which is also a right noetherian ring. It is well-known that the right Krull dimension of $T$ is equal to either r.K.dim. $(R)$ or r.K.dim. $(R)+1$, but the exact value of r.K.dim. $(T)$ has been calculated only in some extreme cases $[1,7]$ and in the case of a commutative noetherian coefficient ring [6]. Here we develop a formula for r.K.dim.( $T)$ whenever $R$ is right noetherian with finite right Krull dimension, that generalizes the results in both [7] and [6]. The formula that we obtain gives the Krull dimension of $T$ in terms of K.dim. $\left(A \otimes_{R} T\right)$ and the "height" of $A$, where $A$ ranges over the simple right $R$-modules. The Krull dimension of $A \otimes_{R} T$, which is either 0 or 1 , may be determined by a criterion of Hodges and McConnell [7]. The "height" of $A$ generalizes the idea of the height of a maximal ideal in a commutative ring, and is given by the length of the longest sequence $A=A_{0}, A_{1}, \ldots, A_{n}$ of "clean" modules (i.e., each $A_{l}$ is critical and each $A_{i} \otimes_{R} T$ is critical as well) such that $A_{i}$ is a minor subfactor of $A_{i+1}$ and $A_{n}$ is a subfactor of $R$. In the case that $R$ is a fully bounded noetherian ring these notions reduce to more familiar concepts, and we are able to recover the same formula for r.K.dim. $(T)$ as that provided by Hodges [6] for commutative noetherian coefficient rings.

The pattern of our results, and the line of proof that we follow, are parallel to our earlier work on the corresponding problem of the Krull dimension of a differential operator ring over a right noetherian ring with a derivation $[3,4]$. In the skew-Laurent case, an extra step is sometimes required, for certain graded module arguments must be carried out over the skew-polynomial ring $R[\theta ; \sigma]$ and the results then transferred to the skew-Laurent ring $R\left[\theta, \theta^{-1} ; \sigma\right]$.

All rings in this paper are associative with unit. In dealing with modules over a skew-Laurent extension of a ring $R$ with an automorphism $\sigma$, some results must be expressed in terms of semilinear isomorphisms rather than in terms of isomorphisms. To be specific, consider right 
$R$-modules $A$ and $B$, and an integer $n$. A $\sigma^{n}$-semilinear homomorphism from $A$ to $B$ is any abelian group homomorphism $f: A \rightarrow B$ such that $f(a r)=f(a) \sigma^{n}(r)$ for all $a \in A$ and $r \in R$. (In the terminology of [7], such a map is called a $\sigma^{n}$-twisted $R$-module map.) We may construct a category whose objects are all right $R$-modules and whose morphisms are all $\sigma^{n}$-semilinear homomorphisms between them, for arbitrary $n$. A morphism in this category is an isomorphism if and only if it is bijective, a monomorphism if and only if it is injective, and an epimorphism if and only if it is surjective. Thus there is no problem in using the terms $\sigma^{n}$-semilinear isomorphism (monomorphism, epimorphism). We define $A \approx_{\sigma} B$ if and only if there is a $\sigma^{n}$-semilinear isomorphism of $A$ onto $B$ for some $n$, and we define $A \lesssim_{\sigma} B$ if and only if there is a $\sigma^{n}$-semilinear monomorphism of $A$ into $B$ for some $n$. Note that a $\sigma^{n}$-semilinear monomorphism of $A$ into $B$ induces an embedding of the lattice of submodules of $A$ into the lattice of submodules of $B$, whence K.dim.( $A)$ $\leq \mathrm{K} \cdot \operatorname{dim} .(B)$ (provided $A$ and $B$ have Krull dimension). In particular, if $A \approx_{\sigma} B$, then K.dim. $(A)=\mathrm{K} \cdot \operatorname{dim} .(B)$.

Given a ring $R$ with an automorphism $\sigma$, the skew-polynomial extension $S=R[\theta ; \sigma]$ is the free left $R$-module with basis $\left\{1, \theta, \theta^{2}, \ldots\right\}$, given a ring structure by the relations $\theta^{n} r=\sigma^{n}(r) \theta^{n}$ for $r \in R$ and $n \in \mathbf{N}$, together with the usual multiplication in $R$. This ring is also referred to as an Ore extension of $R$. The skew-Laurent extension $T=R\left[\theta, \theta^{-1} ; \sigma\right]$ is the corresponding ring, which, as a left $R$-module, is free with basis

$$
\left\{1, \theta, \theta^{-1}, \theta^{2}, \theta^{-2}, \ldots\right\} \text {. }
$$

Alternatively, the multiplicative set $\left\{1, \theta, \theta^{2}, \ldots\right\}$ is a right and left Ore set in $S$, and $T$ may be identified with the Ore localization of $S$ at this multiplicative set. It follows that $T$ is flat as a right or a left $S$-module.

We abbreviate the above by writing "let $T=R\left[\theta, \theta^{-1} ; \sigma\right]$ be a skew-Laurent extension" to stand for "let $R$ be a ring, let $\sigma$ be an automorphism of $R$, and let $T=R\left[\theta, \theta^{-1} ; \sigma\right]$ ". We similarly use the abbreviation "let $S=R[\theta ; \sigma]$ be a skew-polynomial extension". The reader should be forewarned that although the skew-polynomial construction can be carried out with a ring equipped with a ring endomorphism, in this paper we will always assume that we are dealing with an automorphism.

Given a right $R$-module $A$, the nonzero elements of either $A \otimes_{R} S$ or $A \otimes_{R} T$ can be written uniquely in the form

$$
x=\left(a_{m} \otimes \theta^{m}\right)+\left(a_{m+1} \otimes \theta^{m+1}\right)+\cdots+\left(a_{n-1} \otimes \theta^{n-1}\right)+\left(a_{n} \otimes \theta^{n}\right),
$$


where the $a_{i} \in A$ and $a_{m}, a_{n} \neq 0$. (Of course, for $x \in A \otimes_{R} S$ we must have $m \geq 0$.) The $a_{i}$ are called the coefficients of $x$, and $a_{n}$ is the leading coefficient of $x$. The integer $n$ is the degree of $x$, denoted $\operatorname{deg}(x)$, and the positive integer $n-m+1$ is the length of $x$. The element 0 is defined to have degree $-\infty$, leading coefficient 0 , and length 0 . There is a natural ascending filtration

$$
X_{0} \leq X_{1} \leq X_{2} \leq \cdots
$$

on $A \otimes_{R} S$ given by the $R$-submodules

$$
X_{n}=\left\{x \in A \otimes_{R} S \mid \operatorname{deg}(x) \leq n\right\},
$$

and $X_{0} \cong A$ while $X_{n} / X_{n-1} \approx_{\mathrm{o}} A$ for all $n=1,2, \ldots$. Similarly, there is a doubly infinite filtration

$$
\cdots \leq Y_{-2} \leq Y_{-1} \leq Y_{0} \leq Y_{1} \leq Y_{2} \leq \cdots
$$

on $A \otimes_{R} T$ given by the $R$-submodules

$$
Y_{n}=\left\{y \in A \otimes_{R} T \mid \operatorname{deg}(y) \leq n\right\},
$$

and $Y_{0} / Y_{-1} \cong A$ while $Y_{n} / Y_{n-1} \approx{ }_{\sigma} A$ for all $n= \pm 1, \pm 2, \ldots$.

The natural basis $\left\{1, \theta, \theta^{2}, \ldots\right\}$ for $S$ as a free left $R$-module is a subset of the corresponding basis for $T$ as a free left $R$-module, so that ${ }_{R} S$ is a direct summand of ${ }_{R} T$. Hence, the inclusion map $S \rightarrow T$ induces an embedding $A \otimes_{R} S \rightarrow A \otimes_{R} T$, and we may identify $A \otimes_{R} S$ with its image in $A \otimes_{R} T$. In particular, when writing

$$
x=\left(a_{0} \otimes 1\right)+\left(a_{1} \otimes \theta\right)+\cdots+\left(a_{n} \otimes \theta^{n}\right)
$$

with $a_{0}, \ldots, a_{n} \in A$, we need not specify whether we are viewing $x$ as an element of $A \otimes_{R} S$ or as an element of $A \otimes_{R} T$.

For any $T$-submodule $B$ of $A \otimes_{R} T$, we write $\lambda(B)$ to denote the set of leading coefficients of elements of $B$. The set $\lambda(B)$ is an $R$-submodule of A.

We use Krull dimension for noncommutative rings in the sense of Rentschler and Gabriel [9], and the reader is referred to the monograph [5] for the basic properties of Krull dimension. When $A$ is a noetherian right $R$-module, then $A \otimes_{R} S$ is a noetherian right $S$-module and $A \otimes_{R} T$ is a noetherian right $T$-module, and the Krull dimensions of $A \otimes_{R} S$ and $A \otimes_{R} T$ equal either K.dim. $(A)$ or K.dim. $(A)+1$. These results are proved by easy graded module and faithful flatness arguments. (E.g., see [10, Lemma 1].) In particular, r.K.dim.(T) equals either r.K.dim. $(R)$ or r.K.dim. $(R)+1$. 
I. Submodules of induced modules. In calculating the Krull dimensions of induced modules over a skew-Laurent extension $T=$ $R\left[\theta, \theta^{-1} ; \sigma\right]$, that is, modules of the form $A \otimes_{R} T$, we shall need to consider the kinds of $R$-modules that can occur as subfactors, in addition to studying descending chains of $T$-submodules. We consider various cases that arise, in the following series of results. In each case, we proceed by first deriving the corresponding result for induced modules $A \otimes_{R} S$ over the skew-polynomial extension $S=R[\theta ; \sigma]$.

Lemma 1.1. Let $S=R[\theta ; \sigma]$ be a skew-polynomial extension, let $A$ be a right $R$-module, and let $B$ be a nonzero $S$-submodule of $A \otimes_{R} S$. Then there exists a nonzero right $R$-module $C$ such that $C \lesssim_{\sigma} A$ and $C \otimes_{R} S$ embeds in $B$.

Proof. Choose a nonzero element $x \in B$ of minimal degree $n$, and write

$$
x=\left(x_{0} \otimes 1\right)+\left(x_{1} \otimes \theta\right)+\cdots+\left(x_{n} \otimes \theta^{n}\right)
$$

where each $x_{i} \in A$ and $x_{n} \neq 0$. Set

$$
I=\left\{r \in R \mid x_{n} \sigma^{n}(r)=0\right\} .
$$

If $r \in I$, then $x r$ is an element of $B$ with degree less than $n$, and so $x r=0$. Thus $x I=0$ and $x I S=0$.

Consider any $s$ in $S-I S$. Write

$$
s=t+\left(s_{0}+s_{1} \theta+\cdots+s_{k} \theta^{k}\right)
$$

where $t \in I S$, each $s_{i} \in R$, and $s_{k} \notin I$. Since $x t=0$, the coefficient of $\theta^{n+k}$ in $x s$ in $x_{n} \sigma^{n}\left(s_{k}\right)$. This coefficient is nonzero because $x_{k} \notin I$, and so $x s \neq 0$.

Thus $I S=\operatorname{ann}_{S}(x)$. Now if $C=R / I$, then

$$
C \otimes_{R} S \cong S / I S \cong x S \leq B .
$$

The rule $r \mapsto x_{n} \sigma^{n}(r)$ defines a $\sigma^{n}$-semilinear homomorphism from $R$ to $A$ with kernel $I$, and hence $C \lesssim_{\sigma} A$.

Although the skew-Laurent analogue of Lemma 1.1 is easily obtained as a corollary of the lemma, by a direct argument we can obtain a stronger result in the skew-Laurent case.

LEMMA 1.2. Let $T=R\left[\theta, \theta^{-1} ; \sigma\right]$ be a skew-Laurent extension, let $A$ be a right $R$-module, and let $B$ be a nonzero $T$-submodule of $A \otimes_{R} T$. Then $A$ contains a nonzero submodule $C$ such that $C \otimes_{R} T$ embeds in $B$. 
Proof. Choose a nonzero element $x \in B$ of minimal length $k$. Replacing $x$ by $x \theta^{m}$ for a suitable integer $m$, we may assume that $x$ has the form

$$
x=\left(x_{0} \otimes 1\right)+\left(x_{1} \otimes \theta\right)+\cdots+\left(x_{k-1} \otimes \theta^{k-1}\right)
$$

where each $x_{i} \in A$ and $x_{0}, x_{k-1} \neq 0$. Set $I=\operatorname{ann}_{R}\left(x_{0}\right)$. If $r \in I$, then $x r$ is an element of $B$ with length less than $k$, and so $x r=0$. Thus $x I=0$ and $x I T=0$.

Consider any $t$ in $T-I T$. Write

$$
t=u+\left(t_{n} \theta^{n}+t_{n+1} \theta^{n+1}+\cdots+t_{p} \theta^{p}\right)
$$

where $u \in I T$, each $t_{i} \in R$, and $t_{n} \notin I$. Since $x u=0$, the coefficient of $\theta^{n}$ in $x t$ is $x_{0} t_{n}$. This coefficient is nonzero because $t_{n} \notin I$, and so $x t \neq 0$.

Thus $I T=\operatorname{ann}_{T}(x)$. Set $C=x_{0} R$. Then $C$ is a nonzero submodule of $A$, and

$$
C \otimes_{R} T \cong(R / I) \otimes_{R} T \cong T / I T \cong x T \leq B .
$$

Corollary 1.3. [6, Lemma 2.3] Let $T=R\left[\theta, \theta^{-1} ; \sigma\right]$ be a skewLaurent extension. If $A$ is a compressible right $R$-module, then $A \otimes_{R} T$ is a compressible right $T$-module.

Proof. If $B$ is a nonzero $T$-submodule of $A \otimes_{R} T$, then by Lemma 1.2 there exists a nonzero submodule $C \leq A$ such that $C \otimes_{R} T$ embeds in $B$. Since $A$ is compressible, $A$ embeds in $C$, and then $A \otimes_{R} T$ embeds in $C \otimes_{R} T$, because $T$ is a flat left $R$-module. Therefore $A \otimes_{R} T$ embeds in $B$.

Proposition 1.4. Let $S=R[\theta ; \sigma]$ be a skew-polynomial extension, let $A$ be a noetherian right $R$-module, and let $I, J$ be $S$-submodules of $A \otimes_{R} S$ such that $I \leq J$. Suppose that $B$ is a nonzero noetherian right $R$-module such that $B \otimes_{R} S$ is isomorphic to an $S$-module subfactor of $J / I$. Then there exists a nonzero subfactor $C$ of $\lambda(J) / \lambda(I)$ such that $C \lesssim_{\sigma} B$.

Proof. By enlarging $I$ and reducing $J$, if necessary, we may assume that $J / I$ is isomorphic to $B \otimes_{R} S$. As an $R$-module, $B \otimes_{R} S$ is the union of an ascending chain of submodules with the successive subfactors semilinearly isomorphic to $B$. Hence, in $A \otimes_{R} S$ there exist $R$-submodules

$$
I=B_{0}<B_{1}<B_{2}<\cdots<J
$$

with $\cup B_{i}=J$ and each $B_{i+1} / B_{i} \approx_{\sigma} B$. Note that since $B$ is noetherian, each $B_{i} / I$ is a noetherian $R$-module. 
For each nonnegative integer $n$, set

$$
\begin{gathered}
I_{n}=\{x \in I \mid \operatorname{deg}(x) \leq n\}, \\
\lambda_{n}(I)=\left\{y \in A \mid y \text { is the leading coefficient of some } x \in I_{n}\right\}
\end{gathered}
$$

and define $J_{n}$ and $\lambda_{n}(J)$ in the same manner. Then $I_{0} \leq I_{1} \leq \cdots$ is an ascending chain of $R$-submodules of $A \otimes_{R} S$ and $\lambda_{0}(I) \leq \lambda_{1}(I) \leq \cdots$ is an ascending chain of submodules of $A$, and similarly for the $J_{n}$ and the $\lambda_{n}(J)$. Since $A$ is noetherian, there exists a positive integer $p$ such that $\lambda_{n}(I)=\lambda_{p}(I)$ and $\lambda_{n}(J)=\lambda_{p}(J)$ for all $n \geq p$, so that $\lambda_{n}(I)=\lambda(I)$ and $\lambda_{n}(J)=\lambda(J)$ for all $n \geq p$. Thus for $n \geq p$ we obtain the following semilinear isomorphism of $R$-modules:

$$
\begin{aligned}
\left(I+J_{n}\right) /\left(I+J_{n-1}\right) & \cong J_{n} /\left(J_{n} \cap\left(I+J_{n-1}\right)\right)=J_{n} /\left(I_{n}+J_{n-1}\right) \\
& \approx_{\sigma} \lambda_{n}(J) / \lambda_{n}(I)=\lambda(J) / \lambda(I) .
\end{aligned}
$$

As the $R$-module $\left\{x \in A \otimes_{R} S \mid \operatorname{deg}(x) \leq p\right\}$ is noetherian, so is $J_{p}$, and hence $\left(I+J_{p}\right) / I$ is noetherian. Consequently, there exists a positive integer $q$ such that

$$
\left(B_{q+1} / I\right) \cap\left(\left(I+J_{p}\right) / I\right)=\left(B_{q} / I\right) \cap\left(\left(I+J_{p}\right) / I\right) .
$$

Thus $B_{q+1} \cap J_{p} \leq B_{q}$. Set $D=B_{q+1}+J_{p}$ and $E=B_{q}+J_{p}$, so that $D$ and $E$ are $R$-submodules of $A \otimes_{R} S$ with $E \leq D$ and

$$
D / E \cong B_{q+1} /\left(B_{q+1} \cap\left(B_{q}+J_{p}\right)\right)=B_{q+1} / B_{q} \approx_{\sigma} B .
$$

Also, since $D / I$ is a finitely generated $R$-submodule of $J / I$ (because $B_{q+1} / I$ and $J_{p}$ are each finitely generated), $D \leq I+J_{n}$ for some $n>p$.

On applying the Schreier Refinement Theorem to the two chains of $R$-modules

$$
\begin{gathered}
I+J_{p} \leq E<D \leq I+J_{n}, \\
I+J_{p} \leq I+J_{p+1} \leq \cdots \leq I+J_{n},
\end{gathered}
$$

we see that some nonzero submodule $F$ of $D / E$ is isomorphic to a subfactor $G$ of one of the modules $\left(I+J_{m+1}\right) /\left(I+J_{m}\right)$, where $p \leq m<n$. Using the semilinear isomorphisms (1) and (2), we conclude that there is a nonzero subfactor $C$ of $\lambda(J) / \lambda(I)$ such that

$$
C \approx_{\sigma} G \cong F \lesssim_{\sigma} B
$$

COROLlaRY 1.5. Let $T=R\left[\theta, \theta^{-1} ; \sigma\right]$ be a skew-Laurent extension, let $A$ be a noetherian right $R$-module, and let $I, J$ be T-submodules of $A \otimes_{R} T$ such that $I \leq J$. Suppose that $B$ is a nonzero noetherian right $R$-module such 
that $B \otimes_{R} T$ is isomorphic to a $T$-module subfactor of $J / I$. Then there exists a nonzero subfactor $C$ of $\lambda(J) / \lambda(I)$ such that $C \lesssim_{\sigma} B$.

Proof. We may assume that $J / I \cong B \otimes_{R} T$, in which case there exists a $T$-module epimorphism $f: J \rightarrow B \otimes_{R} T$ such that $\operatorname{ker}(f)=I$. Set $S=$ $R[\theta ; \sigma]$, and set

$$
I_{0}=I \cap\left(A \otimes_{R} S\right) \text { and } J_{0}=J \cap\left(A \otimes_{R} S\right) .
$$

Note that $\lambda\left(I_{0}\right)=\lambda(I)$ and $\lambda\left(J_{0}\right)=\lambda(J)$. Also, note that

$$
I_{0} T=I<J=J_{0} T
$$

whence $J_{0} / I_{0} \neq 0$.

As $A$ is a noetherian $R$-module, $A \otimes_{R} S$ is a noetherian $S$-module, and hence $J_{0}$ is a finitely generated $S$-module. Choose $S$-module generators $x_{1}, \ldots, x_{k}$ for $J_{0}$, and then choose a negative integer $n$ such that each $f\left(x_{i}\right)$ lies in $\left(B \otimes_{R} S\right) \theta^{n}$. Hence, $f\left(J_{0}\right)$ is contained in $\left(B \otimes_{R} S\right) \theta^{n}$. Since $I_{0}$ equals the kernel of the restriction of $f$ to $J_{0}$, it follows that $J_{0} / I_{0}$ is isomorphic to an $S$-submodule of $\left(B \otimes_{R} S\right) \theta^{n}$.

Make the abelian group $B$ into a new right $R$-module $B^{\prime}$ by using the $R$-module multiplication $*$ under which $b * r=b \sigma^{n}(r)$ for all $b \in B$ and all $r \in R$. Then the rule

$$
b \otimes \theta^{i} \mapsto b \otimes \theta^{i+n}
$$

defines an $S$-module isomorphism of $B^{\prime} \otimes_{R} S$ onto $\left(B \otimes_{R} S\right) \theta^{n}$. Thus $J_{0} / I_{0}$ is isomorphic to a nonzero $S$-submodule of $B^{\prime} \otimes_{R} S$.

By Lemma 1.1, there exists a nonzero right $R$-module $D$ such that $D \lesssim_{\sigma} B^{\prime}$ and $D \otimes_{R} S$ embeds in $J_{0} / I_{0}$. Since $B^{\prime} \approx_{\sigma} B$, we also have $D \lesssim_{\sigma} B$. Applying Proposition 1.4, we obtain a nonzero subfactor $C$ of $\lambda\left(J_{0}\right) / \lambda\left(I_{0}\right)$ such that $C \lesssim_{\sigma} D$. Then $C$ is a subfactor of $\lambda(J) / \lambda(I)$, and $C \lesssim_{\sigma} B$ as well.

Proposition 1.6. Let $S=R[\theta ; \sigma]$ be a skew-polynomial extension, and let $A$ be an $\alpha$-critical noetherian right $R$-module, for some ordinal $\alpha$. Let

$$
A \otimes_{R} S \geq B_{1} \geq B_{2} \geq \cdots \geq B>0
$$

where the $B_{i}$ are $R$-submodules of $A \otimes_{R} S$ and $B$ is a nonzero $S$-submodule of $A \otimes_{R} S$. Then there exists a positive integer $p$ such that for any integer $j \geq p$, all finitely generated $R$-module subfactors of $B_{j} / B_{j+1}$ have Krull dimension less than $\alpha$.

Proof. Set $A_{n}=\left\{x \in A \otimes_{R} S \mid \operatorname{deg}(x) \leq n\right\}$ for each $n=0,1,2, \ldots$. Then each $A_{n+1} / A_{n} \approx_{\sigma} A$, whence $A_{n+1} / A_{n}$ is $\alpha$-critical, and so 
K.dim. $\left(A_{n}\right)=\alpha$ for all $n$. Note that any finitely generated $R$-submodule of $A \otimes_{R} S$ is contained in some $A_{n}$ and so is noetherian. Thus all finitely generated $R$-module subfactors of $A \otimes_{R} S$ have Krull dimension.

Choose a nonzero element $b \in B$ with positive degree $t$. For $n=t$, $t+1, \ldots$, we must have

$$
A_{n} \cap\left(B+A_{n-1}\right)>A_{n-1},
$$

because $b \theta^{n-t}$ lies in $\left(A_{n} \cap B\right)-A_{n-1}$. Thus $A_{n} /\left(A_{n} \cap\left(B+A_{n-1}\right)\right)$ is a proper factor of $A_{n} / A_{n-1}$. In addition,

$$
\left(B+A_{n}\right) /\left(B+A_{n-1}\right) \cong A_{n} /\left(A_{n} \cap\left(B+A_{n-1}\right)\right),
$$

so that $\left(B+A_{n}\right) /\left(B+A_{n-1}\right)$ is isomorphic to a proper factor of $A_{n} / A_{n-1}$. Hence,

$$
\text { K.dim. }\left(\left(B+A_{n}\right) /\left(B+A_{n-1}\right)\right)<\alpha
$$

for each $n \geq t$. As $\left(A \otimes_{R} S\right) /\left(B+A_{t}\right)$ is the union of the submodules

$$
\left(B+A_{t+1}\right) /\left(B+A_{t}\right) \leq\left(B+A_{t+2}\right) /\left(B+A_{t}\right) \leq \cdots,
$$

it follows that all finitely generated $R$-module subfactors of

$$
\left(A \otimes_{R} S\right) /\left(B+A_{t}\right)
$$

have Krull dimension less than $\alpha$.

In the $R$-module $\left(A \otimes_{R} S\right) / B$, consider the following two chains of submodules:

$$
\begin{gathered}
\left(A \otimes_{R} S\right) / B \geq\left(B+A_{t}\right) / B \geq 0, \\
B_{1} / B \geq B_{2} / B \geq B_{3} / B \geq \cdots .
\end{gathered}
$$

We may use [3, Proposition 3.2(b)] to obtain a common refinement of these chains. Namely, there exist $R$-submodules

$$
\begin{gathered}
A \otimes_{R} S \geq V_{01} \geq V_{02} \geq \cdots \geq B+A_{t} \\
B+A_{t} \geq V_{11} \geq V_{12} \geq \cdots \geq B \\
B_{j}=W_{0 j} \geq W_{1 j} \geq W_{2 j}=B_{j+1}
\end{gathered}
$$

(for $j=1,2, \ldots$ ) such that $V_{i j} / V_{i, j+1} \cong W_{i j} / W_{l+1, j}$ for all $i, j$. Now

$$
\mathrm{K} \cdot \operatorname{dim} .\left(\left(B+A_{t}\right) / B\right) \leq \mathrm{K} \cdot \operatorname{dim} .\left(A_{t}\right)=\alpha .
$$

Hence, there exists a positive integer $p$ such that

$$
\text { K.dim. }\left(V_{1 j} / V_{1, j+1}\right)<\alpha
$$


for all $j \geq p$. Consequently, for $j \geq p$ the module $W_{1 j} / W_{2 j}$ is a noetherian $R$-module with

$$
\text { K.dim. }\left(W_{1 j} / W_{2 j}\right)<\alpha .
$$

Since $W_{0 j} / W_{1 j}$ is isomorphic to $V_{0 j} / V_{0, j+1}$, which is a subfactor of $\left(A \otimes_{R} S\right) /\left(B+A_{t}\right)$, all finitely generately subfactors of $W_{0 j} / W_{1 j}$ have Krull dimension less than $\alpha$. Therefore for $j \geq p$, every finitely generated subfactor of $B_{j} / B_{j+1}$ has Krull dimension less than $\alpha$.

Corollary 1.7. Let $T=R\left[\theta, \theta^{-1} ; \sigma\right]$ be a skew-Laurent extension, and let $A$ be an $\alpha$-critical noetherian right $R$-module, for some ordinal $\alpha$. Let

$$
A \otimes_{R} T \geq B_{1} \geq B_{2} \geq \cdots \geq B>0
$$

be a chain of nonzero $T$-submodules of $A \otimes_{R} T$. Then there exists a positive integer $p$ such that for any integer $j \geq p$, all finitely generated $R$-module subfactors of $B_{j} / B_{j+1}$ have Krull dimension less than $\alpha$.

Proof. Set $S=R[\theta ; \sigma]$, set $C_{j}=B_{j} \cap\left(A \otimes_{R} S\right)$ for each positive integer $j$, and set $C=B \cap\left(A \otimes_{R} S\right)$. Then we obtain a chain of $S$-submodules

$$
A \otimes_{R} S \geq C_{1} \geq C_{2} \geq \cdots \geq C>0 .
$$

By Proposition 1.6, there exists a positive integer $p$ such that for $j \geq p$, all finitely generated $R$-module subfactors of $C_{j} / C_{j+1}$ have Krull dimension less than $\alpha$.

Suppose, for some $j \geq p$, that $B_{J} / B_{j+1}$ has a finitely generated $R$-module subfactor with Krull dimension at least $\alpha$ (or with no Krull dimension). Then there exists a finitely generated $R$-submodule $E \leq B$, such that $E /\left(E \cap B_{j+1}\right)$ either has no Krull dimension or else has Krull dimension at least $\alpha$. Choose a positive integer $n$ such that $E \theta^{n} \leq A \otimes_{R} S$, and note that $E \approx_{\sigma} E \theta^{n}$. As observed in the proof of Proposition 1.6, all finitely generated $R$-submodules of $A \otimes_{R} S$ are noetherian. Thus $E \theta^{n}$ is noetherian, and so $E$ is noetherian, whence $E /\left(E \cap B_{j+1}\right)$ has Krull dimension. Now

$$
\text { K.dim. }\left(E /\left(E \cap B_{j+1}\right)\right) \geq \alpha .
$$

Observe that $E \theta^{n} \leq B_{j} \cap\left(A \otimes_{R} S\right)=C_{j}$, and so

$$
E \theta^{n} \cap C_{j+1}=E \theta^{n} \cap B_{J+1}=\left(E \cap B_{j+1}\right) \theta^{n} .
$$

Hence, multiplication by $\theta^{n}$ induces a $\sigma^{-n}$-semilinear isomorphism

$$
E /\left(E \cap B_{j+1}\right) \approx_{\sigma} E \theta^{n} /\left(E \theta^{n} \cap C_{j+1}\right),
$$


and consequently K.dim. $\left(E \theta^{n} /\left(E \theta^{n} \cap C_{j+1}\right)\right) \geq \alpha$. On the other hand,

$$
\text { K.dim. }\left(E \theta^{n} /\left(E \theta^{n} \cap C_{j+1}\right)\right)<\alpha
$$

because $E \theta^{n} /\left(E \theta^{n} \cap C_{j+1}\right)$ is isomorphic to a finitely generated $R$-submodule of $C_{j} / C_{j+1}$, and we have reached a contradiction.

Therefore for $j \geq p$, all finitely generated $R$-module subfactors of $B_{j} / B_{j+1}$ must have Krull dimension less than $\alpha$.

II. Clean modules. This section is devoted to the basic induction step needed in calculating the Krull dimensions of induced modules over a skew-Laurent extension $T=R\left[\theta, \theta^{-1} ; \sigma\right]$. When $A$ is a critical noetherian right $R$-module, the aim is to describe the Krull dimension of $A \otimes_{R} T$ in terms of the Krull dimensions of the modules $A^{\prime} \otimes_{R} T$ as $A^{\prime}$ ranges over critical subfactors of $A$ with lower Krull dimension than $A$. If $A$ happens to be compressible, then by Corollary $1.3, A \otimes_{R} T$ is compressible, and hence critical. In particular, this holds if $R$ is commutative, or if $A$ is simple. (That $A \otimes_{R} T$ is critical when $A$ is simple is also shown in [7, Corollary 4.8].) However, in general $A \otimes_{R} T$ need not be critical, as shown by an example at the end of the section. Thus we restrict attention to those critical $R$-modules $A$ for which $A \otimes_{R} T$ is critical, as follows.

Definition. Let $R \subseteq T$ be rings. As in [3, Section IV], we define a $T$-clean right $R$-module to be any critical right $R$-module. $A$ such that $A \otimes_{R} T$ is a critical right $T$-module. We shall sometimes refer just to clean $R$-modules if no ambiguity is likely to arise. Note that all clean modules are nonzero, because they are critical. Also, note that if $T$ is flat as a left $R$-module (as in the case that $T$ is a skew-Laurent extension of $R$ ), then any nonzero submodule of a $T$-clean right $R$-module is $T$-clean. The next lemina guarantees the existence of an abundance of clean modules in the skew-Laurent context.

LEMMA 2.1. Let $T=R\left[\theta, \theta^{-1} ; \sigma\right]$ be a skew-Laurent extension. If $A$ is a nonzero noetherian right $R$-module, then $A$ contains a $T$-clean submodule.

Proof. Since $A \otimes_{R} T$ is a noetherian $T$-module, it has Krull dimension, and so it contains a critical $T$-submodule $B$. By Lemma 1.2, $A$ contains a nonzero submodule $C$ such that $C \otimes_{R} T$ embeds in $B$. Since $A$ is noetherian, $C$ has Krull dimension, and hence $C$ contains a critical $R$-submodule $D$. Now $D \otimes_{R} T$ embeds in $B$, and so $D \otimes_{R} T$ is a critical $T$-module. Therefore $D$ is a $T$-clean $R$-submodule of $A$. 
For one technical step in our procedure, we require a notion of height 1 for clean modules, as used in [3, Section IV]. Recall that a minor subfactor of a module $A$ is any submodule of a proper factor of $A$.

Definition. Let $R \subseteq T$ be rings, and let $A, B$ be $T$-clean noetherian right $R$-modules. Define $h_{T}(A: B)=1$ if $A$ is isomorphic to a minor subfactor of $B$ but no nonzero submodule of $A$ is isomorphic to a minor subfactor of a $T$-clean minor subfactor of $B$. We drop the subscript $T$ in cases in which no ambiguity is likely to arise. Note that if $h_{T}(A: B)=1$, then $h_{T}\left(A^{\prime}: B\right)=1$ for all nonzero $T$-clean submodules $A^{\prime}$ of $A$. In case $h_{T}(A: B)=1$ does not hold, we write $h_{T}(A: B) \neq 1$.

LEMMA 2.2. Let $T=R\left[\theta, \theta^{-1} ; \sigma\right]$ be a skew-Laurent extension, let $B$ be a $T$-clean noetherian right $R$-module, and let

$$
B \geq C_{1} \geq C_{2} \geq \cdots \geq C>0
$$

be a chain of submodules of $B$. Then there exists a positive integer $p$ such that for all integers $j \geq p$, there are no $T$-clean subfactors $X$ of $C_{J} / C_{J+1}$ satisfying $h_{T}(X: B)=1$.

Proof. As every nonzero noetherian right $R$-module contains a $T$-clean submodule (Lemma 2.1), the lemma is a special case of [3, Lemma 4.3].

LEMMA 2.3. Let $S=R[\theta ; \sigma]$ be a skew-polynomial extension, let $B$ be a noetherian right $R$-module, and let $D$ be a nonzero $S$-module subfactor of $B \otimes_{R} S$. Then there exists a nonzero subfactor $C$ of $B$ such that $C \lesssim_{\sigma} D_{R}$.

Proof. Set $B_{n}=\left\{x \in B \otimes_{R} S \mid \operatorname{deg}(x) \leq n-2\right\}$ for all positive integers $n$, and write $D=E / F$ for some $S$-submodules $F<E$ in $B \otimes_{R} S$. Consider the following chains of $R$-submodules:

$$
\begin{aligned}
& 0 \leq F<E \leq B \otimes_{R} S, \\
& 0=B_{1} \leq B_{2} \leq \cdots .
\end{aligned}
$$

We may use [3, Proposition 3.2(a)] to obtain a common refinement of these chains. Namely, there exist $R$-submodules

$$
\begin{aligned}
& 0=V_{01} \leq V_{02} \leq \cdots \leq F \\
& F=V_{11} \leq V_{12} \leq \cdots \leq E \\
& E=V_{21} \leq V_{22} \leq \cdots \leq B \otimes_{R} S \\
& B_{J}=W_{0 J} \leq W_{1 J} \leq W_{2 J} \leq W_{3 J}=B_{J+1}
\end{aligned}
$$


(for $j=1,2, \ldots$ ) such that $V_{i, j+1} / V_{l j} \cong W_{i+1, j} / W_{i j}$ for all $i, j$, and also

$$
\bigcup_{j=1}^{\infty} V_{1 j}=E \text {. }
$$

Let $k$ be the least positive integer such that $V_{1 k}>F$, and set $C^{\prime}=V_{1 k} / F$. Then $C^{\prime}$ is a nonzero $R$-submodule of $D$ such that

$$
C^{\prime}=V_{1 k} / V_{1, k-1} \cong W_{2, k-1} / W_{1, k-1},
$$

so that $C^{\prime}$ is isomorphic to a subfactor of $B_{k} / B_{k-1}$. Since $B_{k} / B_{k-1} \approx_{\sigma} B$, there exists a nonzero subfactor $C$ of $B$ such that $C \approx_{\sigma} C^{\prime}$, and hence $C \lesssim_{\sigma} D$.

Recall that if $T=R\left[\theta, \theta^{-1} ; \sigma\right]$ is a skew-Laurent extension, and if $M$ and $N$ are right $R$-modules such that $M \approx_{\sigma} N$, then $M \otimes_{R} T \cong N \otimes_{R} T$. Namely, if $f$ is a $\sigma^{k}$-semilinear isomorphism of $M$ onto $N$, for some integer $k$, then there is a $T$-module isomorphism

$$
g: M \otimes_{R} T \rightarrow N \otimes_{R} T
$$

such that $g(m \otimes t)=f(m) \otimes \theta^{k} t$ for all $m \in M$ and $t \in T$.

COROLlary 2.4. Let $T=R\left[\theta, \theta^{-1} ; \sigma\right]$ be a skew-Laurent extension, let $B$ be a noetherian right $R$-module, and let $D$ be a nonzero $T$-module subfactor of $B \otimes_{R} T$. Then there exist a T-clean subfactor $A$ of $B$ and $a$ $T$-clean $R$-submodule $A^{\prime} \leq D$ such that $A \approx_{\sigma} A^{\prime}$.

Proof. Write $D=E / F$ for some $T$-submodules $F<E$ in $B \otimes_{R} T$. Let $S=R[\theta ; \sigma]$, and set

$$
E_{0}=E \cap\left(B \otimes_{R} S\right) \text { and } F_{0}=F \cap\left(B \otimes_{R} S\right) .
$$

Since $F_{0} T=F<E=E_{0} T$, we see that $E_{0} / F_{0}$ is a nonzero $S$-module subfactor of $B \otimes_{R} S$. Note that $E_{0} / F_{0}$ embeds in $D$.

By Lemma 2.3, there exists a nonzero subfactor $A$ of $B$ such that $A \lesssim_{\sigma}\left(E_{0} / F_{0}\right)_{R}$. Since $A$ has $T$-clean submodules (Lemma 2.1), there is no loss of generality in assuming that $A$ itself is $T$-clean. As $E_{0} / F_{0}$ embeds in $D$, there is an $R$-submodule $A^{\prime} \leq D$ such that $A \approx_{\sigma} A^{\prime}$; since $A \otimes_{R} T \cong$ $A^{\prime} \otimes_{R} T$, we conclude that $A^{\prime}$ is $T$-clean.

We are now in a position to derive an expression for the Krull dimension of an induced module $B \otimes_{R} T$ over a skew-Laurent extension $T=R\left[\theta, \theta^{-1} ; \sigma\right]$, at least when $B$ is clean, in terms of the Krull dimensions of the modules $A \otimes_{R} T$, where $A$ runs through the clean minor subfactors of $B$. 
Proposition 2.5. Let $T=R\left[\theta, \theta^{-1} ; \sigma\right]$ be a skew-Laurent extension, and let $B$ be a $T$-clean noetherian right $R$-module with finite Krull dimension. If $B$ is not simple, then

$$
\text { K.dim. }\left(B \otimes_{R} T\right)=\max \left\{\mathbf{K} \cdot \operatorname{dim} .\left(A \otimes_{R} T\right) \mid A \in \mathbb{Q}\right\}+1,
$$

where $\mathbb{Q}$ is the family of $T$-clean minor subfactors of $B$.

Proof. Since $B$ is not simple, it has some nonzero minor subfactors, and so $\mathcal{Q}$ is nonempty, because of Lemma 2.1. Set $\beta=$ K.dim.( $B)$. Since $\beta$ is finite, $\mathrm{K} \cdot \operatorname{dim} .\left(B \otimes_{R} T\right)$ is finite, and hence the value

$$
\alpha=\max \left\{\mathrm{K} \cdot \operatorname{dim} .\left(A \otimes_{R} T\right) \mid A \in \mathbb{Q}\right\}
$$

is really a maximum (rather than a supremum). Choose $A \in \mathbb{Q}$ with

$$
\text { K.dim. }\left(A \otimes_{R} T\right)=\alpha .
$$

Then $A \otimes_{R} T$ is isomorphic to a minor subfactor of $B \otimes_{R} T$, and so

$$
\text { K.dim. }\left(B \otimes_{R} T\right)>\alpha,
$$

because $B \otimes_{R} T$ is critical. Thus K.dim. $\left(B \otimes_{R} T\right) \geq \alpha+1$.

If $\mathrm{K} . \operatorname{dim} .\left(B \otimes_{R} T\right)>\alpha+1$, then

$$
\text { K.dim. }\left(\left(B \otimes_{R} T\right) / C\right)>\alpha
$$

for some nonzero $T$-submodule $C$ of $B \otimes_{R} T$. Hence, there exists a chain of $T$-submodules

$$
B \otimes_{R} T \geq C_{1} \geq C_{2} \geq \cdots \geq C>0
$$

such that K.dim. $\left(C_{j} / C_{j+1}\right) \geq \alpha$ for infinitely many $j$. After refining this chain, we may assume that each $C_{j} / C_{j+1}$ is critical. By Corollary 1.7, there exists a positive integer $p$ such that for any $j \geq p$, all finitely generated $R$-module subfactors of $C_{j} / C_{j+1}$ have Krull dimension less than $\beta$.

Inside $B$, there is a chain of $R$-submodules

$$
B \geq \lambda\left(C_{1}\right) \geq \lambda\left(C_{2}\right) \geq \cdots \geq \lambda(C)>0 .
$$

By Lemma 2.2, there exists a positive integer $q$ such that for all $j \geq q$, there are no clean subfactors $X$ of $\lambda\left(C_{j}\right) / \lambda\left(C_{J+1}\right)$ satisfying $h(X: B)=1$.

Choose $m \geq \max \{p, q\}$ such that $\operatorname{K} \cdot \operatorname{dim} .\left(C_{m} / C_{m+1}\right) \geq \alpha$. By Corollary 2.4, there exist a clean subfactor $A$ of $B$ and a clean $R$-submodule $A^{\prime}$ of $C_{m} / C_{m+1}$ such that $A \approx_{\sigma} A^{\prime}$. Since $m \geq p$, we have K.dim. $\left(A^{\prime}\right)<$ $\beta$, and so K.dim. $(A)<\beta$. Thus $A$ must be a minor subfactor of $B$. Then $A \in \mathbb{Q}$ and

K.dim. $\left(A \otimes_{R} T\right) \leq \alpha$. 
Since $A \approx_{\sigma} A^{\prime}$, we have $A \otimes_{R} T \cong A^{\prime} \otimes_{R} T$, and hence

$$
\text { K.dim. }\left(A^{\prime} \otimes_{R} T\right) \leq \alpha .
$$

Now set $E=A^{\prime} T$, which is a nonzero $T$-submodule of $C_{m} / C_{m+1}$, and note that

$$
\mathrm{K} \cdot \operatorname{dim} .(E)=\mathrm{K} \cdot \operatorname{dim} .\left(C_{m} / C_{m+1}\right) \geq \alpha,
$$

because $C_{m} / C_{m+1}$ is critical.

The module $E$ is a homomorphic image of the critical module $A^{\prime} \otimes_{R} T$. As

$$
\mathrm{K} \cdot \operatorname{dim} .(E) \geq \alpha \geq \mathrm{K} \cdot \operatorname{dim} .\left(A^{\prime} \otimes_{R} T\right),
$$

we see that $E$ cannot be a proper homomorphic image of $A^{\prime} \otimes_{R} T$. Thus $E \cong A^{\prime} \otimes_{R} T$, and

$$
\mathrm{K} \cdot \operatorname{dim} .(E)=\mathrm{K} \cdot \operatorname{dim} .\left(A^{\prime} \otimes_{R} T\right)=\alpha .
$$

Now $A^{\prime} \otimes_{R} T$ is isomorphic to a submodule of $C_{m} / C_{m+1}$. By Corollary 1.5, there exists a nonzero subfactor $X$ of $\lambda\left(C_{m}\right) / \lambda\left(C_{m+1}\right)$ such that $X \lesssim_{\sigma} A^{\prime}$. Since $X$ is a subfactor of $\lambda\left(C_{m}\right) / \lambda\left(C_{m+1}\right)$, it is also a minor subfactor of $B$. Because of Lemma 2.1 , there is no loss of generality in assuming that $X$ is clean. Hence, $h(X: B) \neq 1$, because $m \geq q$. Thus there exist a nonzero submodule $Y \leq X$ and a clean minor subfactor $G$ of $B$ such that $Y$ is isomorphic to a minor subfactor of $G$. Then $G \in Q$, and $Y \otimes_{R} T$ is isomorphic to a minor subfactor of the critical module $G \otimes_{R} T$, whence

$$
\text { K.dim. }\left(Y \otimes_{R} T\right)<\mathrm{K} \cdot \operatorname{dim} .\left(G \otimes_{R} T\right) \leq \alpha .
$$

On the other hand, $Y \approx_{\sigma} Y^{\prime}$ for some submodule $Y^{\prime} \leq A^{\prime}$, and so $Y \otimes_{R} T$ $\cong Y^{\prime} \otimes_{R} T$. Since $Y^{\prime} \otimes_{R} T$ is isomorphic to a nonzero submodule of the critical module $A^{\prime} \otimes_{R} T$, it follows that

$$
\mathrm{K} \cdot \operatorname{dim} .\left(Y \otimes_{R} T\right)=\mathrm{K} \cdot \operatorname{dim} .\left(A^{\prime} \otimes_{R} T\right)=\alpha,
$$

which is a contradiction.

Therefore K.dim. $\left(B \otimes_{R} T\right)=\alpha+1$.

We conclude this section with an example of an unclean critical module, to show the necessity of using clean modules rather than critical modules in our results.

EXAMPLE 2.6. There exists a skew-Laurent extension $T=R\left[\theta, \theta^{-1} ; \sigma\right]$ of a right and left noetherian domain $R$ such that $R$ has a 1-critical cyclic right module which is not $T$-clean. 
Proof. Choose a field $K$ of characteristic zero, let $t, x$ be independent indeterminates, and set

$$
R_{0}=K(t)\left[x, x^{-1}\right] .
$$

Define commuting $K$-algebra automorphisms $\sigma_{1}$ and $\sigma_{2}$ on $R_{0}$ so that $\sigma_{1}(t)=2 t$ and $\sigma_{1}(x)=x$ while $\sigma_{2}(t)=t$ and $\sigma_{2}(x)=2 x$. Now set

$$
R=R_{0}\left[\theta_{1}, \theta_{1}^{-1} ; \sigma_{1}\right],
$$

which is a right and left noetherian domain. Since $\sigma_{1}$ and $\sigma_{2}$ commute, we may extend $\sigma_{2}$ to an automorphism of $R$ such that $\sigma_{2}\left(\theta_{1}\right)=\theta_{1}$. Set

$$
T=R\left[\theta_{2}, \theta_{2}^{-1} ; \sigma_{2}\right]=R_{0}\left[\theta_{1}^{ \pm 1}, \theta_{2}^{ \pm 1} ; \sigma_{1}, \sigma_{2}\right] .
$$

It is easily checked that no maximal ideal of $R_{0}$ is invariant under any nonzero power of $\sigma_{2}$. Hence, by [7, Lemma 3.4], all simple $R_{0}$-modules tensor up to simple modules over $R_{0}\left[\theta_{2}, \theta_{2}^{-1} ; \sigma_{2}\right]$. Thus $R_{0}\left[\theta_{2}, \theta_{2}^{-1} ; \sigma_{2}\right]$ has right Krull dimension 1 , by [7, Theorem 6.1], and consequently r.K.dim. $(T) \leq 2$.

We construct a 1-critical cyclic right $R$-module $A$, built as a non-split extension of a 1-critical module $C$ by a simple module $B$, such that $A \otimes_{R} T$ is not critical, which occurs because $B \otimes_{R} T$ and $C \otimes_{R} T$ each have Krull dimension 1.

First, set $B=R /(x+t) R$. Since $(x+t) R_{0}$ is a maximal ideal of $R_{0}$ that is not invariant under any nonzero power of $\sigma_{1}$, it follows from [7, Lemma 3.4] that $(x+t) R$ is a maximal right ideal of $R$. Thus $B$ is a simple $R$-module. On the other hand,

$$
\theta_{1} \sigma_{2}((x+t) R)=\theta_{1}(2 x+t) R=(2 x+2 t) \theta_{1} R=(x+t) R
$$

and $\theta_{1} \in R-(x+t) R$, and hence [7, Lemma 3.5] shows that $B \otimes_{R} T$ is a non-artinian $T$-module. As $B \otimes_{R} T$ is compressible (Corollary 1.3) and therefore critical, it must be 1-critical.

Next, set $C=R /\left(\theta_{1}-1\right) R$. Since $R=R_{0} \oplus\left(\theta_{1}-1\right) R$ as right $R_{0^{-}}$ modules, we may identify $C$ with $R_{0}$, made into a right $R$-module with a module multiplication $*$ such that $r * \theta_{1}=\sigma_{1}^{-1}(r)$ for all $r \in R_{0}$. Thus the lattice of $R$-submodules of $C$ is isomorphic to the lattice of $\sigma_{1}$-invariant ideals of $R_{0}$. Each of the ideals in the chain

$$
(x-1) R_{0}>(x-1)^{2} R_{0}>(x-1)^{3} R_{0}>\cdots
$$

is $\sigma_{1}$-invariant, and so $C$ is not artinian. However, $C$ is 1 -critical as an $R_{0}$-module. Therefore $C$ must also be a 1-critical $R$-module. Since $C \otimes_{R} T$ is isomorphic to $T /\left(\theta_{1}-1\right) T$, which is a proper factor of the critical 
module $T_{T}$, we have

$$
\text { K.dim. }\left(C \otimes_{R} T\right)<\text { r.K.dim. }(T) \leq 2 .
$$

On the other hand, K.dim. $\left(C \otimes_{R} T\right) \geq \mathrm{K} \cdot \operatorname{dim} .(C)=1$, and hence

$$
\text { K.dim. }\left(C \otimes_{R} T\right)=1 \text {. }
$$

Finally, set $A=R /(x+t)\left(\theta_{1}-1\right) R$, which is an extension of $C$ by $B$. If this extension splits, then by [2, Lemma 2.9] there exist elements $u, v \in R$ such that

$$
u(x+t)+\left(\theta_{1}-1\right) v=1 .
$$

Write $u=\left(\theta_{1}-1\right) r+r_{0}$ for some $r \in R$ and $r_{0} \in R_{0}$. Then

$$
\left(\theta_{1}-1\right)(v+r(x+t))=1-r_{0}(x+t)
$$

and so $r_{0}(x+t)=1$, which is impossible. Thus the extension is not split.

Therefore $A$ is a non-split extension of the 1-critical module $C$ by the simple module $B$, whence $A$ is a 1 -critical right $R$-module. On the other hand, $A \otimes_{R} T$ is an extension of $C \otimes_{R} T$ by $B \otimes_{R} T$, each of which has Krull dimension 1 , and hence $A \otimes_{R} T$ is not critical. Therefore $A$ is not $T$-clean.

III. Krull dimension formulas. The formula given in Proposition 2.5 is designed for use in an inductive procedure, by which the Krull dimension of an induced module $B \otimes_{R} T$ over a skew-Laurent extension $T=R\left[\theta, \theta^{-1} ; \sigma\right]$ may be computed in terms of the Krull dimensions of the modules $M \otimes_{R} T$ where $M$ runs through the simple subfactors of $B$. In order to keep track of the number of steps in which Proposition 2.5 is used, a notion of height for simple modules is required, as in [3, Section V]. A height value for non-simple clean modules is not required.

Definition. Let $R \subseteq T$ be rings, let $M$ be a simple right $R$-module, and let $B$ be an arbitrary right $R$-module. Then $h_{T}(M: B)$ is defined to be the supremum of those nonnegative integers $n$ for which there exists a sequence $M=A_{0}, A_{1}, \ldots, A_{n}$ of $T$-clean right $R$-modules such that $A_{i}$ is isomorphic to a minor subfactor of $A_{l+1}$ for $i=0,1, \ldots, n-1$ while $A_{n}$ is isomorphic to a subfactor (not necessarily minor) of $B$. Given such a sequence,

K.dim. $\left(A_{n}\right)>\mathrm{K} \cdot \operatorname{dim} .\left(A_{n-1}\right)>\cdots>\mathrm{K} \cdot \operatorname{dim} .\left(A_{1}\right)>\mathrm{K} \cdot \operatorname{dim} .\left(A_{0}\right)=0$, because each $A_{l}$ is critical, and hence K.dim. $(B) \geq n$ if K.dim.(B) exists. Thus if $B$ has Krull dimension, then $h_{T}(M: B) \leq \mathrm{K}$.dim. $(B)$. Note that $h_{T}(M: B) \geq 0$ only if $M$ is $T$-clean and isomorphic to a subfactor of $B$; otherwise, $h_{T}(M: B)=-\infty$. 
TheOREM 3.1. Let $T=R\left[\theta, \theta^{-1} ; \sigma\right]$ be a skew-Laurent extension, and let $B$ be a nonzero noetherian right $R$-module with finite Krull dimension. Then

$$
\text { K.dim. }\left(B \otimes_{R} T\right)=\max \left\{\mathbf{K} \cdot \operatorname{dim} .\left(M \otimes_{R} T\right)+h_{T}(M: B) \mid M \in \mathfrak{\Re}\right\},
$$
where $\mathfrak{N}$ is the set of simple subfactors of $B$.

Proof. Set $\beta=\mathrm{K}$.dim.( $B)$. If $\beta=0$, then $B$ has submodules

$$
0=B_{0}<B_{1}<B_{2}<\cdots<B_{k}=B
$$

such that each $B_{i} / B_{i-1}$ is simple, and each simple subfactor of $B$ is isomorphic to some $B_{i} / B_{i-1}$. Note that $h_{T}(M: B)=0$ for all $M \in \Re$. Thus

$$
\begin{aligned}
\mathrm{K} \cdot \operatorname{dim} .\left(B \otimes_{R} T\right) & =\max \left\{\mathrm{K} \cdot \operatorname{dim} .\left(\left(B_{i} / B_{i-1}\right) \otimes_{R} T\right) \mid i=1, \ldots, k\right\} \\
& =\max \left\{\mathrm{K} \cdot \operatorname{dim} \cdot\left(M \otimes_{R} T\right) \mid M \in \mathfrak{N}\right\} \\
& =\max \left\{\mathrm{K} \cdot \operatorname{dim} \cdot\left(M \otimes_{R} T\right)+h_{T}(M: B) \mid M \in \Re\right\}
\end{aligned}
$$

in this case.

Now let $\beta>0$ and assume that the result holds for noetherian $R$-modules of Krull dimension less than $\beta$. Set

$$
\mu=\max \left\{\mathrm{K} \cdot \operatorname{dim} .\left(M \otimes_{R} T\right)+h_{T}(M: B) \mid M \in \mathfrak{N}\right\} .
$$

If $M \in \mathfrak{T}$ and $h_{T}(M: B)=n$, then there exist clean right $R$-modules $M=A_{0}, A_{1}, \ldots, A_{n}$ such that $A_{i}$ is isomorphic to a minor subfactor of $A_{i+1}$ for $i=0,1, \ldots, n-1$ while $A_{n}$ is isomorphic to a subfactor of $B$. Then each $A_{i} \otimes_{R} T$ is isomorphic to a minor subfactor of the critical module $A_{l+1} \otimes_{R} T$, whence

K.dim. $\left(A_{n} \otimes_{R} T\right)>$ K.dim. $\left(A_{n-1} \otimes_{R} T\right)$

$$
>\cdots>\mathrm{K} \cdot \operatorname{dim} \cdot\left(A_{0} \otimes_{R} T\right)=\mathrm{K} \cdot \operatorname{dim} \cdot\left(M \otimes_{R} T\right) .
$$

As $A_{n} \otimes_{R} T$ is isomorphic to a subfactor of $B \otimes_{R} T$, we obtain

$\mathrm{K} \cdot \operatorname{dim} .\left(B \otimes_{R} T\right) \geq \mathrm{K} \cdot \operatorname{dim} .\left(A_{n} \otimes_{R} T\right) \geq \mathrm{K} \cdot \operatorname{dim} .\left(M \otimes_{R} T\right)+n$

$$
=\mathrm{K} \cdot \operatorname{dim} \cdot\left(M \otimes_{R} T\right)+h_{T}(M: B) \text {. }
$$

Thus K.dim. $\left(B \otimes_{R} T\right) \geq \mu$.

Use Lemma 2.1 to choose submodules

$$
0=C_{0}<C_{1}<C_{2}<\cdots<C_{k}=B
$$


such that each $C_{i} / C_{i-1}$ is clean. Any simple subfactor $M$ of $C_{l} / C_{l-1}$ is also a simple subfactor of $B$, and

$$
h_{T}\left(M: C_{i} / C_{i-1}\right) \leq h_{T}(M: B),
$$

whence

$$
\max \left\{\mathrm{K} \cdot \operatorname{dim} .\left(M \otimes_{R} T\right)+h_{T}\left(M: C_{i} / C_{l-1}\right) \mid M \in \Re_{l}\right\} \leq \mu,
$$

where $\mathfrak{R}_{i}$ is the set of simple subfactors of $C_{l} / C_{l-1}$. Thus we need only show that our Krull dimension formula holds for each $C_{t} / C_{t-1}$. Hence, there is no loss of generality in assuming that $B$ is clean.

By Proposition 2.5, there exists a clean minor subfactor $A$ of $B$ such that

$$
\text { K.dim. }\left(B \otimes_{R} T\right)=\mathrm{K} \cdot \operatorname{dim} .\left(A \otimes_{R} T\right)+1 .
$$

Since $B$ is critical, K.dim. $(A)<\beta$. Then, by the induction hypothesis, there exists a simple subfactor $M$ of $A$ such that

$$
\mathrm{K} \cdot \operatorname{dim} .\left(A \otimes_{R} T\right)=\mathrm{K} \cdot \operatorname{dim} .\left(M \otimes_{R} T\right)+h_{T}(M: A) .
$$

Consequently,

K.dim. $\left(B \otimes_{R} T\right)=\mathrm{K} \cdot \operatorname{dim} .\left(M \otimes_{R} T\right)+h_{T}(M: A)+1$.

In addition, $M \in \Re$, and $h_{T}(M: B) \geq h_{T}(M: A)+1$, because $A$ is a minor subfactor of the clean module $B$. Hence,

$\mathrm{K} \cdot \operatorname{dim} .\left(B \otimes_{R} T\right) \leq \mathrm{K} \cdot \operatorname{dim} .\left(M \otimes_{R} T\right)+h_{T}(M: B) \leq \mu$.

Therefore K.dim. $\left(B \otimes_{R} T\right)=\mu$.

COROLlARY 3.2. Let $R$ be a right noetherian ring with finite right Krull dimension, let $\sigma$ be an automorphism of $R$, and set $T=R\left[\theta, \theta^{-1} ; \sigma\right]$. Then

r.K.dim. $(T)=\max \left\{\mathbf{K} \cdot \operatorname{dim} .\left(M \otimes_{R} T\right)+h_{T}(M: R) \mid M \in \Re\right\}$, where $\mathfrak{N}$ is the family of simple right $R$-modules.

COROLlaRY 3.3. Let $R$ be a right noetherian ring with finite right Krull dimension, let $\sigma$ be an automorphism of $R$, and set $T=R\left[\theta, \theta^{-1} ; \sigma\right]$. Then

$$
\text { r.K.dim. }(T)=\text { r.K.dim. }(R)
$$

unless there exists a simple right $R$-module $M$ such that

$$
\mathrm{K} . \operatorname{dim} .\left(M \otimes_{R} T\right)=1 \text { and } h_{T}(M: R)=\mathrm{r} . \mathrm{K} \cdot \operatorname{dim} .(R) \text {, }
$$

in which case

$$
\text { r.K.dim. }(T)=\text { r.K.dim. }(R)+1 \text {. }
$$


COROLlARY 3.4. Let $R$ be a right noetherian ring with finite right Krull dimension, let $\sigma$ be an automorphism of $R$, and set $T=R\left[\theta, \theta^{-1} ; \sigma\right]$. Then

$$
\text { r.K.dim. }(T)=\text { r.K.dim. }(R)
$$

unless there exists a maximal right ideal $J$ of $R$ such that

$$
h_{T}(R / J: R)=\text { r.K.dim. }(R)
$$

and $x \sigma^{n}(J) \subseteq J$ for some element $x \in R-J$ and some nonzero integer $n$. In this latter case,

$$
\text { r.K.dim. }(T)=\text { r.K.dim. }(R)+1 .
$$

Proof. Corollary 3.3 and [7, Lemmas 3.4, 3.5].

Two cases of Corollary 3.3 were obtained previously by Hodges and McConnell, namely, that

$$
\text { r.K.dim. }(T)=\text { r.K.dim. }(R)+1
$$

when all simple right $R$-modules tensor up to 1-critical $T$-modules [7, Theorem 5.1], and that

$$
\text { r.K.dim. }(T)=\text { r.K.dim. }(R)
$$

when all simple right $R$-modules tensor up to simple $T$-modules [7, Theorem 6.1]. The commutative version of Corollary 3.4 was proved by Hodges [6, Theorem 2.8]. In that result, for a maximal ideal $J$ of $R$ the value $h_{T}(R / J: R)$ is of course replaced by the ordinary height of $J$. However,

$$
h_{T}(R / J: R)=h t(J) \text {, }
$$

as we shall see in Proposition 4.2, and hence Hodges' result may be viewed as a special case of our result.

IV. Fully bounded noetherian coefficient rings. In this section, we specialize our results to the case of a skew-Laurent extension $T=$ $R\left[\theta, \theta^{-1} ; \sigma\right]$ where $R$ is a fully bounded noetherian ring. In this case the values $h_{T}(M: R)$ for simple $R$-modules $M$ may be replaced by the heights of maximal two-sided ideals of $R$, and our Krull dimension formula for $T$ reduces to exactly the same result as that obtained by Hodges in the case that $R$ is commutative [6, Theorem 2.8].

LEMMA 4.1. Let $T=R\left[\theta, \theta^{-1} ; \sigma\right]$ be a skew-Laurent extension of a right noetherian ring $R$. Let $M$ be a simple right $R$-module, set $P=\operatorname{ann}_{R}(M)$ and assume that $R / P$ is a simple artinian ring. Then $\mathrm{K} \cdot \operatorname{dim} .\left(M \otimes_{R} T\right)=1$ if and only if $P$ is invariant under some nonzero power of $\sigma$. 
Proof. We may assume that $M=R / J$ for some maximal right ideal $J$ of $R$ that contains $P$.

If K.dim. $(T / J T)=1$, then by [7, Lemma 3.4], $x \sigma^{n}(J) \subseteq J$ for some $x$ in $R-J$ and some nonzero integer $n$. Then $\sigma^{n}(J)$ is a maximal right ideal of $R$ such that $R / \sigma^{n}(J) \cong R / J$, and hence $P \subseteq \sigma^{n}(J)$. For any $r \in R$ and $p \in P$, we have $\sigma^{n}(r) p \in P$, whence $r \sigma^{-n}(p) \in J$. Thus

$$
\sigma^{-n}(P) \subseteq \operatorname{ann}_{R}(R / J)=P .
$$

Conversely, assume that $\sigma^{n}(P) \subseteq P$ for some nonzero integer $n$. Since $P$ is a maximal two-sided ideal of $R$, so is $\sigma^{n}(P)$, and hence $\sigma^{n}(P)=P$. Then $P \subseteq \sigma^{n}(J)$, from which it follows that

$$
\operatorname{ann}_{R}\left(R / \sigma^{n}(J)\right)=P,
$$

and consequently $R / \sigma^{n}(J) \cong R / J$, because $R / P$ is a simple artinian ring. Hence, $x \sigma^{n}(J) \subseteq J$, for some $x$ in $R-J$. By [7, Lemma 3.5], $\mathrm{K} \cdot \operatorname{dim} .(T / J T)=1$.

Proposition 4.2. Let $T=R\left[\theta, \theta^{-1} ; \sigma\right]$ be a skew-Laurent extension of a fully bounded noetherian ring $R$. Let $M$ be a simple right $R$-module, and let $P=\operatorname{ann}_{R}(M)$. Then $h_{T}(M: R)=\mathrm{ht}(P)$.

Proof. The proof of [3, Proposition 6.2] may be repeated here.

THEOREM 4.3. Let $R$ be a fully bounded noetherian ring with finite right Krull dimension, let $\sigma$ be an automorphism of $R$, and set $T=R\left[\theta, \theta^{-1} ; \sigma\right]$. Then

$$
\text { r.K.dim. }(T)=\text { r.K.dim. }(R)
$$

unless there exists a maximal two-sided ideal $P$ of $R$ such that $\mathrm{ht}(P)=$ r.K.dim. $(R)$ and $P$ is invariant under some nonzero power of $\sigma$. In this latter case,

$$
\text { r.K.dim. }(T)=\text { r.K.dim. }(R)+1 \text {. }
$$

Proof. Corollary 3.3, Lemma 4.1, Proposition 4.2.

B. Noncommutative COEFFicients; ITERATEd EXTENSIONS

The purpose of this part of the paper is to derive some of the basic induction steps needed in calculating the Krull dimension of a skewLaurent extension formed from a coefficient ring equipped with several commuting automorphisms. For these induction arguments, the commutativity of the automorphisms is irrelevant, and so we proceed in the context 
of iterated skew-Laurent extensions. In addition to providing the key induction steps for the final part of the paper, these results also allow us to derive a criterion that determines when an iterated skew-Laurent extension can achieve its maximum possible Krull dimension, analogous to the criterion derived for iterated differential operator ring extensions in [2].

V. Iterated skew-Laurent extensions. Let $R$ be a ring, and let $u$ be a positive integer. A $u$-fold iterated skew-Laurent extension of $R$ is any ring of the form

$$
T=R\left[\theta_{1}, \theta_{1}^{-1} ; \sigma_{1}\right]\left[\theta_{2}, \theta_{2}^{-1} ; \sigma_{2}\right] \cdots\left[\theta_{u}, \theta_{u}^{-1}, \sigma_{u}\right],
$$

where $\sigma_{1}$ is an automorphism of $R$, and $\sigma_{i}$, for $i=2, \ldots, u$, is an automorphism of the ring

$$
R\left[\theta_{1}, \theta_{1}^{-1} ; \sigma_{1}\right]\left[\theta_{2}, \theta_{2}^{-1} ; \sigma_{2}\right] \cdots\left[\theta_{i-1}, \theta_{i-1}^{-1} ; \sigma_{l-1}\right] .
$$

We abbreviate the expression for $T$ to $T=R\left[\theta_{1}^{ \pm 1}, \ldots, \theta_{u}^{ \pm 1}\right]$. Such extensions are of course studied by procedures following induction on $u$, starting from results in the case that $u=1$. For instance, if $A$ is any noetherian right $R$-module, then $A \otimes_{R} T$ is a noetherian right $T$-module, and

$$
\mathrm{K} \cdot \operatorname{dim} .(A) \leq \mathrm{K} \cdot \operatorname{dim} .\left(A \otimes_{R} T\right) \leq \mathrm{K} \cdot \operatorname{dim} .(A)+u \text {. }
$$

LEMMA 5.1. Let $T=R\left[\theta, \theta^{-1} ; \sigma\right]$ be a skew-Laurent extension, and let $A$ be a right $T$-module. Then there exists a short exact sequence

$$
0 \rightarrow A \otimes_{R} T \rightarrow A \otimes_{R} T \rightarrow A \rightarrow 0
$$

of right T-modules.

Proof. Let $g: A \otimes_{R} T \rightarrow A$ be the map given by the $T$-module multiplication on $A$, so that $g(a \otimes t)=a t$ for all $a \in A$ and $t \in T$. Then $g$ is a $T$-module epimorphism.

Next, define an additive map $f: A \otimes_{R} T \rightarrow A \otimes_{R} T$ so that

$$
f\left(a \otimes \theta^{n}\right)=\left(a \theta \otimes \theta^{n-1}\right)-\left(a \otimes \theta^{n}\right)
$$

for all $a \in A$ and $n \in \mathbf{Z}$. We shall show that $f$ is a $T$-module homomorphism. Clearly, $f(x \theta)=f(x) \theta$ and $f\left(x \theta^{-1}\right)=f(x) \theta^{-1}$ for all $x$ in $A \otimes_{R} T$. 
Also, if $a \in A, n \in \mathbf{Z}, r \in R$, then

$$
\begin{aligned}
\left(f\left(a \otimes \theta^{n}\right)\right) r & =\left(a \theta \otimes \theta^{n-1} r\right)-\left(a \otimes \theta^{n} r\right) \\
& =\left(a \theta \sigma^{n-1}(r) \otimes \theta^{n-1}\right)-\left(a \sigma^{n}(r) \otimes \theta^{n}\right) \\
& =\left(a \sigma^{n}(r) \theta \otimes \theta^{n-1}\right)-\left(a \sigma^{n}(r) \otimes \theta^{n}\right) \\
& =f\left(a \sigma^{n}(r) \otimes \theta^{n}\right)=f\left(\left(a \otimes \theta^{n}\right) r\right) .
\end{aligned}
$$

It follows that $f$ is a $T$-module homomorphism.

Clearly, $f$ is a monomorphism, and $g f=0$. Given $x$ in $\operatorname{ker}(g)$, we show that $x$ is in the image of $f$, by induction on the length of $x$. If $x$ has length at most 1 , write $x=a \otimes \theta^{n}$ for some $a \in A$ and $n \in \mathbf{Z}$. Then $a \theta^{n}=0$ and so $a=0$, whence $x=0=f(0)$.

If $x$ has length greater than 1, write

$$
x=\sum_{i=m}^{n} a_{i} \otimes \theta^{i}
$$

with $m<n$, all $a_{i} \in A$, and $a_{m}, a_{n} \neq 0$. Set

$$
y=x+f\left(a_{n} \otimes \theta^{n}\right)=x+\left(a_{n} \theta \otimes \theta^{n-1}\right)-\left(a_{n} \otimes \theta^{n}\right),
$$

which has shorter length than $x$, and notice that $y \in \operatorname{ker}(g)$. Thus $y$ is in the image of $f$, and hence so is $x$.

Therefore the sequence is exact.

Proposition 5.2. Let $T=R\left[\theta_{1}^{ \pm 1}, \ldots, \theta_{u}^{ \pm 1}\right]$ be an iterated skew-Laurent extension, and let $A$ be a nonzero right $T$-module. If $A$ is noetherian as a right $R$-module, then

$$
\mathrm{K} \cdot \operatorname{dim} .\left(A \otimes_{R} T\right) \geq \mathrm{K} \cdot \operatorname{dim}_{\cdot T}(A)+u .
$$

Proof. Since $A$ is a noetherian right $R$-module, $A \otimes_{R} T$ is a noetherian right $T$-module and so has Krull dimension. First consider the case in which $u=1$, and set $B=A \otimes_{R} T$. In view of Lemma $5.1, B$ has a $T$-submodule $B_{1}$ such that $B_{1} \cong B$ and $B / B_{1} \cong A$. As $B_{1} \cong B$, we may continue this process, obtaining an infinite descending chain of $T$-submodules

$$
A \otimes_{R} T=B>B_{1}>B_{2}>\cdots
$$

with $B_{n} / B_{n+1} \cong A$ for each $n$. Then K.dim. $\left(A \otimes_{R} T\right)>$ K.dim. $T(A)$, completing this case. 
For the general case, let $u>1$ and assume that the result holds for modules over $(u-1)$-fold iterated skew-Laurent extensions. Set $S=$ $R\left[\theta_{1}^{ \pm 1}\right]$, so that $T=S\left[\theta_{2}^{ \pm 1}, \ldots, \theta_{u}^{ \pm 1}\right]$. Since $A$ is noetherian as an $R$-module, it is also noetherian as an $S$-module, and so by the induction hypothesis,

$$
\text { K.dim. }\left(A \otimes_{S} T\right) \geq \mathrm{K} \cdot \operatorname{dim}_{\cdot}(A)+u-1 .
$$

Now by Lemma 5.1 there exists a short exact sequence of right $S$-modules

$$
0 \rightarrow A \otimes_{R} S \rightarrow A \otimes_{R} S \rightarrow A \rightarrow 0,
$$

and since $T$ is a flat left $S$-module, this induces a short exact sequence of right $T$-modules

$$
0 \rightarrow A \otimes_{R} T \rightarrow A \otimes_{R} T \rightarrow A \otimes_{S} T \rightarrow 0 .
$$

It follows that there exists an infinite descending chain of $T$-submodules

$$
A \otimes_{R} T>B_{1}>B_{2}>\cdots
$$

with $B_{n} / B_{n+1} \cong A \otimes_{S} T$ for each $n$. Therefore K.dim. $\left(A \otimes_{R} T\right)>$ K.dim. $\left(A \otimes_{S} T\right)$, and hence

$$
\mathrm{K} \cdot \operatorname{dim} .\left(A \otimes_{R} T\right) \geq \mathrm{K} \cdot \operatorname{dim} \cdot{ }_{T}(A)+u .
$$

In studying the Krull dimensions of modules over iterated skewLaurent extensions, clean modules of course arise again. Here, in order to fit inductive procedures, cleanness must be defined so that criticality is preserved at each iteration, as follows.

Definition. Let $T=R\left[\theta_{1}^{ \pm 1}, \ldots, \theta_{u}^{ \pm 1}\right]$ be a $u$-fold iterated skewLaurent extension, and set $T_{k}=R\left[\theta_{1}^{ \pm 1}, \ldots, \theta_{k}^{ \pm 1}\right]$ for each $k=1, \ldots, u$. A $u$-clean right $R$-module (relative to this extension) is any critical right $R$-module $A$ such that $A \otimes_{R} T_{k}$ is a critical right $T_{k}$-module for each $k=1, \ldots, u$. Note that all compressible noetherian right $R$-modules are $u$-clean, because of Corollary 1.3.

LEMMA 5.3. Let $T=R\left[\theta_{1}^{ \pm 1}, \ldots, \theta_{u}^{ \pm 1}\right]$ be an iterated skew-Laurent extension. If $A$ is a nonzero noetherian right $R$-module, then $A$ contains a $u$-clean submodule.

Proof. The proof is by induction on $u$, the case in which $u=1$ being Lemma 2.1. Thus assume that $u>1$, and that the result holds over $(u-1)$-fold iterated skew-Laurent extensions.

Set $T_{k}=R\left[\theta_{1}^{ \pm 1}, \ldots, \theta_{k}^{ \pm 1}\right]$ for each $k=1, \ldots, u$, and set $S=T_{1}$, so that $T=S\left[\theta_{2}^{ \pm 1}, \ldots, \theta_{u}^{ \pm 1}\right]$. Now $A \otimes_{R} S$ is a nonzero noetherian right 
$S$-module, and hence, by the induction hypothesis, $A \otimes_{R} S$ contains a $(u-1)$-clean submodule $B$. Thus $B \otimes_{S} T_{k}$ is a critical right $T_{k}$-module for each $k=1,2, \ldots, u$. By Lemma $1.2, A$ contains a nonzero submodule $C$ such that $C \otimes_{R} S$ embeds in $B$. Choose a critical submodule $D$ of $C$. For each $k=1, \ldots, u$, we have

$$
D \otimes_{R} T_{k} \cong\left(D \otimes_{R} S\right) \otimes_{S} T_{k},
$$

which embeds in $B \otimes_{S} T_{k}$, and so $D \otimes_{R} T_{k}$ is a critical $T_{k}$-module. Therefore $D$ is a $u$-clean submodule of $A$.

For technical purposes, we again need a notion of height 1, for $u$-clean modules.

Definition. Let $T=R\left[\theta_{1}^{ \pm 1}, \ldots, \theta_{u}^{ \pm 1}\right]$ be an iterated skew-Laurent extension, and let $A, B$ be $u$-clean noetherian right $R$-modules. Define $h_{u}(A: B)=1$ if and only if $A$ is isomorphic to a minor subfactor of $B$ but no nonzero submodule of $A$ is isomorphic to a minor subfactor of a $u$-clean minor subfactor of $B$.

LEMMA 5.4. Let $T=R\left[\theta_{1}^{ \pm 1}, \ldots, \theta_{u}^{ \pm 1}\right]$ be an iterated skew-Laurent extension, let $B$ be a u-clean noetherian right $R$-module, and let

$$
B \geq C_{1} \geq C_{2} \geq \cdots \geq C>0
$$

be a chain of submodules of $B$. Then there exists a positive integer $p$ such that for all integers $j \geq p$, there are no u-clean subfactors $X$ of $C_{j} / C_{j+1}$ satisfying $h_{u}(X: B)=1$.

Proof. The proof is identical to the proof of [3, Lemma 4.3], with " $T$-clean" replaced by " $u$-clean".

Recall from Part A the notation $\lambda(C)$ for the submodule of leading coefficients of a submodule $C$ of an induced module $A \otimes_{R} T$, when $T=R\left[\theta_{1}^{ \pm 1}\right]$ is a 1 -fold skew-Laurent extension.

Proposition 5.5. Let $T=R\left[\theta_{1}^{ \pm 1}, \ldots, \theta_{u}^{ \pm 1}\right]$ be an iterated skew-Laurent extension, let $B$ be a u-clean noetherian right $R$-module with finite Krull dimension, and set

$$
\alpha=\max \left\{\mathrm{K} \cdot \operatorname{dim} .\left(A \otimes_{R} T\right) \mid A \in \mathbb{Q}\right\},
$$

where $\mathbb{Q}$ is the set of $u$-clean minor subfactors of $B$. If

K.dim. $\left(B \otimes_{R} T\right)=\beta>u$,

then $\beta=\alpha+1$. 
Proof. Note that $\beta \geq 2$, and that K.dim. $(B) \geq 1$, so that $B$ is not simple. Thus $B$ has some minor subfactors, and hence $\mathcal{Q}$ is nonempty, by Lemma 5.3. Since K.dim.( $B)$ is finite, $\alpha$ and $\beta$ are both finite.

We proceed by induction on $u$. If $u=1$, then as $B$ is not simple, the result is given by Proposition 2.5.

In the case that $u>1$, first observe that $B \otimes_{R} T$ is critical, because $B$ is $u$-clean. As each $A \otimes_{R} T$ (for $A \in Q$ ) is isomorphic to a minor subfactor of $B \otimes_{R} T$, it follows that

$$
\text { K.dim. }\left(B \otimes_{R} T\right)>\mathrm{K} \cdot \operatorname{dim} .\left(A \otimes_{R} T\right)
$$

for all $A \in \mathbb{Q}$, whence $\beta \geq \alpha+1$.

Set $S=R\left[\theta_{1}^{ \pm 1}\right]$ and $M=B \otimes_{R} S$; then $M$ is a $(u-1)$-clean noetherian right $S$-module and

$$
\mathrm{K} \cdot \operatorname{dim} .\left(M \otimes_{S} T\right)=\beta>u-1 .
$$

Therefore, by the induction hypothesis, there exists a $(u-1)$-clean minor $S$-subfactor $N$ of $M$ such that

$$
\text { K.dim. }\left(N \otimes_{S} T\right)=\beta-1 .
$$

Write $N=C / D$, where $C>D>0$ are nonzero $S$-submodules of $M$.

Since $N$ is a $(u-1)$-clean noetherian right $S$-module with

$$
\text { K.dim. }\left(N \otimes_{S} T\right)=\beta-1>u-1,
$$

we may use the induction hypothesis a second time to obtain a $(u-1)$ clean minor subfactor $N_{1}$ of $N$ such that

$$
\text { K.dim. }\left(N_{1} \otimes_{S} T\right)=\beta-2 \text {. }
$$

Then $N_{1} \cong D_{1} / D_{2}$, where $C \geq D_{1}>D_{2}>D$. Note that $D_{2} / D$ is a $(u-1)$-clean noetherian right $S$-module with

$$
\text { K.dim. }\left(\left(D_{2} / D\right) \otimes_{S} T\right)=\beta-1,
$$

because $\left(D_{2} / D\right) \otimes_{S} T$ is isomorphic to a nonzero submodule of the $(\beta-1)$-critical module $N \otimes_{S} T$. Thus we may repeat the above procedure and find $S$-submodules $D_{2} \geq D_{3}>D_{4}>D$ such that $D_{3} / D_{4}$ is $(u-1)$ clean and

$$
\mathrm{K} . \operatorname{dim} .\left(\left(D_{3} / D_{4}\right) \otimes_{S} T\right)=\beta-2 .
$$

Continue in this way, generating an infinite chain of $S$-submodules

$$
B \otimes_{R} S \geq C \geq D_{1}>D_{2} \geq D_{3}>D_{4} \geq \cdots>D>0
$$

such that each $D_{2 n-1} / D_{2 n}$ is $(u-1)$-clean and

$$
\text { K.dim. }\left(\left(D_{2 n-1} / D_{2 n}\right) \otimes_{S} T\right)=\beta-2 \text {. }
$$


By Corollary 1.7, at most finitely many factors $D_{J} / D_{J+1}$ can have finitely generated $R$-module subfactors with Krull dimensions greater than or equal to K.dim.(B). Also, applying Lemma 5.4 to the chain

$$
B \geq \lambda\left(D_{1}\right) \geq \lambda\left(D_{2}\right) \geq \cdots \geq \lambda(D)>0,
$$

we see that at most finitely many of the factors $\lambda\left(D_{J}\right) / \lambda\left(D_{J+1}\right)$ can have $u$-clean subfactors $X$ satisfying $h_{u}(X: B)=1$. Thus there exists an odd positive integer $k$ such that all finitely generated $R$-module subfactors of $D_{k} / D_{k+1}$ have Krull dimension less than K.dim. $(B)$, and such that all $u$-clean subfactors $X$ of $\lambda\left(D_{k}\right) / \lambda\left(D_{k+1}\right)$ satisfy $h_{u}(X: B) \neq 1$.

Since $k$ is odd,

$$
\mathrm{K} \cdot \operatorname{dim} .\left(\left(D_{k} / D_{k+1}\right) \otimes_{S} T\right)=\beta-2 .
$$

By Corollary 2.4, there exist a nonzero subfactor $E_{0}$ of $B$ and a nonzero $R$-submodule $E_{0}^{\prime}$ of $D_{k} / D_{k+1}$ such that $E_{0} \approx_{\sigma_{1}} E_{0}^{\prime}$. Using Lemma 5.3, choose a $u$-clean submodule $E \leq E_{0}$, and then choose a submodule $E^{\prime} \leq E_{0}^{\prime}$ with $E \approx_{\sigma_{1}} E^{\prime}$. Since $E \otimes_{R} S \cong E^{\prime} \otimes_{R} S$, it follows that $E^{\prime}$ is also $u$-clean. By the choice of $k$, we have

$$
\mathrm{K} \cdot \operatorname{dim} .(E)=\mathrm{K} \cdot \operatorname{dim} .\left(E^{\prime}\right)<\mathrm{K} \cdot \operatorname{dim} .(B),
$$

and hence $E$ must be a minor subfactor of $B$. Set $F=E^{\prime} S$, which is a homomorphic image of $E^{\prime} \otimes_{R} S$, and thus also a homomorphic image of $E \otimes_{R} S$. Now $F \otimes_{S} T$ is isomorphic to a nonzero submodule of $\left(D_{k} / D_{k+1}\right) \otimes_{S} T$, and the latter module is critical, because $D_{k} / D_{k+1}$ is $(u-1)$-clean. Thus $F \otimes_{S} T$ is critical, and

K.dim. $\left(F \otimes_{S} T\right)=\mathrm{K} \cdot \operatorname{dim} .\left(\left(D_{k} / D_{k+1}\right) \otimes_{S} T\right)=\beta-2$.

If $F$ is a proper homomorphic image of $E \otimes_{R} S$, then $F \otimes_{S} T$ is a proper homomorphic image of $E \otimes_{R} T$. Since $E$ is $u$-clean, $E \otimes_{R} T$ is critical, and so

$$
\beta-2=\mathrm{K} . \operatorname{dim} .\left(F \otimes_{S} T\right)<\mathrm{K} \cdot \operatorname{dim} .\left(E \otimes_{R} T\right) \leq \alpha .
$$

Therefore $\beta \leq \alpha+1$ in this case.

Otherwise, $F \cong E \otimes_{R} S$. By Corollary 1.5 , there exists a nonzero subfactor $X$ of $\lambda\left(D_{k}\right) / \lambda\left(D_{k+1}\right)$ such that $X \lesssim_{\sigma_{1}} E$. Because of Lemma 5.3, there is no loss of generality in assuming that $X$ is $u$-clean. By the choice of $k$, we have $h_{u}(X: B) \neq 1$. As $X$ is a minor subfactor of $B$ (because $\lambda\left(D_{k}\right) / \lambda\left(D_{k+1}\right)$ is a minor subfactor of $\left.B\right)$, there must exist a nonzero submodule $Y \leq X$ and a $u$-clean minor subfactor $A$ of $B$, such that $Y$ is isomorphic to a minor subfactor of $A$. Then $Y \otimes_{R} T$ is isomorphic to a minor subfactor of the critical module $A \otimes_{R} T$, and hence

K.dim. $\left(Y \otimes_{R} T\right)<\mathrm{K} . \operatorname{dim} .\left(A \otimes_{R} T\right) \leq \alpha$. 
On the other hand, since $Y \leq X \lesssim_{\sigma_{1}} E$, the module $Y \otimes_{R} T$ embeds in the module $E \otimes_{R} T \cong F \otimes_{S} T$, which is critical. Consequently,

$$
\text { K.dim. }\left(Y \otimes_{R} T\right)=\mathrm{K} \cdot \operatorname{dim} .\left(F \otimes_{S} T\right)=\beta-2 .
$$

Thus $\beta-2<\alpha$, and $\beta \leq \alpha+1$ in this case also.

Therefore $\beta \leq \alpha+1$ in both cases, and hence $\beta=\alpha+1$.

Although Proposition 5.5 does not provide sufficient information to completely determine the Krull dimension of an iterated skew-Laurent extension, it does enable us to derive a criterion that tells when the Krull dimension of an iterated skew-Laurent extension achieves its maximum possible value. This requires a notion of height for simple modules, analogous to that used in §III.

Definition. Let $T=R\left[\theta_{1}^{ \pm 1}, \ldots, \theta_{u}^{ \pm 1}\right]$ be an iterated skew-Laurent extension, and let $M$ be a simple right $R$-module. Define $h_{u}(M)$ (relative to this extension) to be the supremum of those nonnegative integers $k$ for which there is a sequence $M=A_{0}, A_{1}, \ldots, A_{k}$ of $u$-clean noetherian right $R$-modules such that each $A_{l}$ is isomorphic to a minor subfactor of $A_{l+1}$. Note that if the right Krull dimension of $R$ exists, then $h_{u}(M) \leq$ r.K.dim. $(R)$.

THEOREM 5.6. Let $R$ be a right noetherian ring with finite right Krull dimension $k$, and let $T=R\left[\theta_{1}^{ \pm 1}, \ldots, \theta_{u}^{ \pm 1}\right]$ be an iterated skew-Laurent extension of $R$. Then

$$
\text { r.K.dim. }(T)=k+u
$$

if and only if there exists a simple right $R$-module $M$ such that

$$
h_{u}(M)=k \quad \text { and } \quad \mathrm{K} \cdot \operatorname{dim} .\left(M \otimes_{R} T\right)=u .
$$

Proof. First assume that there is a simple right $R$-module $M$ satisfying $h_{u}(M)=k$ and K.dim. $\left(M \otimes_{R} T\right)=u$. Then there exist $u$-clean noetherian right $R$-modules $M=A_{0}, A_{1}, \ldots, A_{k}$ such that each $A_{l}$ is isomorphic to a minor subfactor of $A_{i+1}$. Each $A_{l} \otimes_{R} T$ is a critical right $T$-module, because $A_{l}$ is $u$-clean. In addition, $A_{l} \otimes_{R} T$ is isomorphic to a minor subfactor of $A_{l+1} \otimes_{R} T$, and hence

$$
\mathrm{K} \cdot \operatorname{dim} .\left(A_{\imath+1} \otimes_{R} T\right)>\mathrm{K} \cdot \operatorname{dim} .\left(A_{i} \otimes_{R} T\right) \text {. }
$$

Thus

$$
\mathrm{K} \cdot \operatorname{dim} .\left(A_{k} \otimes_{R} T\right) \geq \mathrm{K} \cdot \operatorname{dim} .\left(A_{0} \otimes_{R} T\right)+k=k+u .
$$


Since r.K.dim. $(T) \leq k+u$ in any case, we conclude that r.K.dim. $(T)=$ $k+u$.

Conversely, assume that r.K.dim. $(T)=k+u$. Because of Lemma 5.3 , there is a finite chain of right ideals from 0 to $R_{R}$ with each successive subfactor being $u$-clean, and at least one of these subfactors must tensor up to a right $T$-module with Krull dimension $k+u$. Thus there exists a $u$-clean noetherian right $R$-module $A_{k}$ such that $A_{k} \otimes_{R} T$ has Krull dimension $k+u$. Note that

$$
\begin{aligned}
k+u & =\mathrm{K} \cdot \operatorname{dim} .\left(A_{k} \otimes_{R} T\right) \leq \mathrm{K} \cdot \operatorname{dim} \cdot\left(A_{k}\right)+u \\
& \leq \mathrm{r} \cdot \mathrm{K} \cdot \operatorname{dim} .(R)+u=k+u,
\end{aligned}
$$

and so K.dim. $\left(A_{k}\right)=k$.

If $k=0$, then $R$ is right artinian, and $A_{k}$ is a simple module. In this case, $h_{u}\left(A_{k}\right)=0$, and we need only take $M=A_{k}$.

Now suppose that $k>0$, whence

$$
\text { K.dim. }\left(A_{k} \otimes_{R} T\right)=k+u>u .
$$

By Proposition 5.5, there exists a $u$-clean minor subfactor $A_{k-1}$ of $A_{k}$ such that

$$
\text { K.dim. }\left(A_{k-1} \otimes_{R} T\right)=k+u-1 .
$$

Continuing in this manner, we obtain $u$-clean noetherian right $R$-modules $A_{k}, A_{k-1}, \ldots, A_{0}$ such that each $A_{i}$ is a minor subfactor of $A_{i+1}$, and

$$
\text { K.dim. }\left(A_{\imath} \otimes_{R} T\right)=i+u
$$

for each $i$. Since K.dim. $\left(A_{i}\right)<\mathrm{K} . \operatorname{dim} .\left(A_{i+1}\right)$ for each $i$, we find that

$$
\text { K.dim. }\left(A_{0}\right)=0 \text {, }
$$

so that $A_{0}$ is a simple right $R$-module. By construction, $h_{u}\left(A_{0}\right) \geq k$, while $h_{u}\left(A_{0}\right) \leq k$ because r.K.dim. $(R)=k$. Thus $h_{u}\left(A_{0}\right)=k$, and the proof is complete (taking $M=A_{0}$ ).

\section{Commutative Coefficients; Multiple Automorphisms}

This part of the paper contains our main result, which provides a precise formula for the Krull dimension of a skew-Laurent extension $T=R\left[\theta_{1}^{ \pm 1}, \ldots, \theta_{u}^{ \pm 1}\right]$ constructed from a commutative noetherian ring $R$ of finite Krull dimension, equipped with commuting automorphisms $\sigma_{1}, \ldots, \sigma_{u}$. As we have seen in Part B, partial information about the Krull dimension of $T$ can be obtained just by iterating the techniques and results of Part A. However, this method alone is not sufficient, for at some stages it is necessary to consider the relationships that may exist among 
the automorphisms. In order to study these relationships, we introduce a concept of "automorphian dimension" of prime ideals of $R$, which plays a role analogous to the differential dimension used in studying the Krull dimensions of differential operator rings [4]. The automorphian dimension of a prime ideal $P$ indicates how large a collection of products of powers of the $\sigma_{i}$ can map $P$ into itself. (In particular, $P$ is invariant under some nonzero power of each of $\sigma_{1}, \ldots, \sigma_{u}$ precisely when the automorphian dimension of $P$ equals $u$.) We compute that the Krull dimension of $T / P T$ is at least as large as the automorphian dimension of $P$, and that if $P$ is a maximal ideal, K.dim. $(T / P T)$ equals this dimension. By an easy argument on chains of prime ideals, it follows that for any prime ideal $P$ of $R$, the number

$$
\text { height }(P)+\text { automorphian dimension }(P)
$$

is a lower bound for the Krull dimension of $T$. Our main theorem shows that K.dim. $(T)$ is actually equal to the maximum value of these lower bounds. The line of proof that we follow is parallel to that used in our earlier work on the corresponding problem of the Krull dimension of a differential operator ring over a commutative noetherian ring with several commuting derivations [4].

Given a ring $R$, and finitely many commuting automorphisms $\sigma_{1}, \ldots, \sigma_{u}$ (not necessarily distinct) of $R$, we may construct the skew-Laurent extension

$$
T=R\left[\theta_{1}, \theta_{1}^{-1}, \ldots, \theta_{u}, \theta_{u}^{-1} ; \sigma_{1}, \ldots, \sigma_{u}\right],
$$

(which we usually abbreviate to $T=R\left[\theta_{1}^{ \pm 1}, \ldots, \theta_{u}^{ \pm 1}\right]$ ), as follows. Additively, $T$ is the abelian group of all Laurent series in independent indeterminates $\theta_{1}, \ldots, \theta_{u}$ over $R$, but multiplication is subject to the requirements that $\theta_{i} \theta_{j}=\theta_{j} \theta_{i}$ for all $i, j$ and that $\theta_{i}{ }^{n} r=\sigma_{i}{ }^{n}(r) \theta_{i}^{n}$ for all $i$, all integers $n$, and all $r \in R$, together with the given multiplication in $R$. This construction may be viewed as a $u$-fold iterated skew-Laurent extension, by extending the automorphisms suitably. Namely, for $t=1, \ldots, u-1$ we may extend $\sigma_{t+1}$ to an automorphism of $R\left[\theta_{1}^{ \pm 1}, \ldots, \theta_{t}^{ \pm 1}\right]$ so that $\sigma_{t+1}\left(\theta_{t}\right)$ $=\theta_{i}$ for $i=1, \ldots, t$, and then $R\left[\theta_{1}^{ \pm 1}, \ldots, \theta_{t+1}^{ \pm 1}\right]$ may be identified with

$$
R\left[\theta_{1}^{ \pm 1}, \ldots, \theta_{t}^{ \pm 1}\right]\left[\theta_{t+1}^{ \pm 1}\right] .
$$

Similarly, $R\left[\theta_{1}^{ \pm 1}, \ldots, \theta_{u}^{ \pm 1}\right]$ may be identified with

$$
R\left[\theta_{1}^{ \pm 1}, \ldots, \theta_{t}^{ \pm 1}\right]\left[\theta_{t+1}^{ \pm 1}, \ldots, \theta_{u}^{ \pm 1}\right] .
$$

In case $R$ is a commutative ring, this skew-Laurent extension $T$ is isomorphic to its opposite ring, since $T$ possesses an involution, namely, 
the anti-automorphism that fixes elements of $R$ and sends each $\theta_{t}$ to $\theta_{t}^{-1}$. Consequently, the right and left Krull dimensions of $T$ (if they exist) are equal, and we may refer just to "the Krull dimension of $T$ ".

VI. Prime and maximal ideals. Our investigation of the Krull dimension of a skew-Laurent extension $T=R\left[\theta_{1}^{ \pm 1}, \ldots, \theta_{u}^{ \pm 1}\right]$ over a commutative noetherian ring $R$ proceeds by considering modules of the form $T / P T$, where $P$ is a prime ideal of $R$. As long as the Krull dimension of $T / P T$ is greater than $u$, Proposition 5.5 may be used to relate K.dim. $(T / P T)$ to the values K.dim. $(T / Q T)$ as $Q$ runs over the prime ideals properly containing $P$. In the case that K.dim. $(T / P T) \leq u$, which in particular holds if $P$ is a maximal ideal, the Krull dimension of $T / P T$ is closely connected to the question of the invariance or non-invariance of $P$ under products of powers of the automorphisms $\sigma_{l}$. To keep track of this invariance, we formulate a notion of independence and dependence of $P$ relative to a set of commuting automorphisms, and we use this notion to define an "automorphian dimension" of $P$ relative to the $\sigma_{i}$. This dimension is then related to the Krull dimension of $T / P T$.

Definition. Let $R$ be a commutative ring, let $\sigma_{1}, \ldots, \sigma_{s}$ be commuting automorphisms of $R$, and let $P$ be a prime ideal of $R$. We say that $\sigma_{1}, \ldots, \sigma_{s}$ are dependent relative to $P$ provided that there exist integers $n(1), \ldots, n(s)$, not all zero, such that

$$
\sigma_{1}^{n(1)} \sigma_{2}^{n(2)} \cdots \sigma_{s}^{n(s)}(P) \subseteq P
$$

If no such integers exist, then we say that $\sigma_{1}, \ldots, \sigma_{s}$ are independent relative to $P$. In particular, if any automorphism in the list $\sigma_{1}, \ldots, \sigma_{s}$ is repeated, then $\sigma_{1}, \ldots, \sigma_{s}$ are dependent relative to $P$, for if $\sigma_{i}=\sigma_{j}$ for some $i \neq j$, then $\sigma_{t}^{1} \sigma_{j}^{-1}(P)=P$.

Definition. Let $R$ be a commutative ring, let $\sigma_{1}, \ldots, \sigma_{u}$ be commuting automorphisms of $R$, and let $P$ be a prime ideal of $R$. The automorphian codimension of $P$ (relative to $\sigma_{1}, \ldots, \sigma_{u}$ ), denoted aut.codim. $(P)$, is the largest nonnegative integer $s$ such that some $s$ of the automorphisms $\sigma_{1}, \ldots, \sigma_{u}$ are independent relative to $P$. In particular, aut.codim. $(P)=0$ if and only if there exist nonzero integers $n(1), \ldots, n(u)$ such that $\sigma_{i}^{n(i)}(P) \subseteq$ $P$ for each $i=1, \ldots, u$. The automorphian dimension of $P$ (relative to $\left.\sigma_{1}, \ldots, \sigma_{u}\right)$, denoted aut.dim. $(P)$, is defined by the rule

aut.dim. $(P)=u-$ aut.codim. $(P)$. 
LEMMA 6.1. Let $R$ be a commutative ring, let $\sigma_{1}, \ldots, \sigma_{s}$ be commuting automorphisms of $R$, and set $T=R\left[\theta_{1}^{ \pm 1}, \ldots, \theta_{s}^{ \pm 1}\right]$. Let $P$ be a prime ideal of $R$, and assume that $\sigma_{1}, \ldots, \sigma_{s}$ are independent relative to $P$. If $J$ is any right ideal of $T$ which properly contains $P T$, then $J \cap R$ properly contains $P$.

Proof. We abbreviate products of powers of the $\theta_{i}$ in the form $\theta^{\alpha}$, where $\alpha$ is an $s$-tuple $(\alpha(1), \ldots, \alpha(s))$ of integers, and $\theta^{\alpha}$ stands for $\theta_{1}^{\alpha(1)} \theta_{2}^{\alpha(2)} \cdots \theta_{s}^{\alpha(s)}$. Similarly, we write $\sigma^{\alpha}$ for $\sigma_{1}^{\alpha(1)} \sigma_{2}^{\alpha(2)} \cdots \sigma_{s}^{\alpha(s)}$.

Choose an element $x$ in $J-P T$ which is a sum of as few monomials as possible. Write

$$
x=\sum_{\alpha \in A} r_{\alpha} \theta^{\alpha}
$$

for some finite subset $A \subseteq \mathbf{Z}^{s}$ and some nonzero elements $r_{\alpha} \in R$. As there is no harm in multiplying $x$ on the right by $\theta^{-\alpha}$ for some choice of $\alpha \in A$, we may assume that the $s$-tuple $0=(0,0, \ldots, 0)$ is in $A$. Note that each $r_{\alpha} \notin P$ (since otherwise $x-r_{\alpha} \theta^{\alpha}$ is an element of $J-P T$ which is a sum of fewer monomials than $x$ ).

Given $p \in P$, we have $p x \in P T$, and so $x p-p x \in J$. In addition,

$$
x p-p x=\sum_{\alpha \in A-\{0\}} r_{\alpha}\left(\sigma^{\alpha}(p)-p\right) \theta^{\alpha},
$$

and hence $x p-p x$ is a sum of fewer monomials than $x$. Consequently, $x p-p x$ lies in $P T$, and so $x p \in P T$. Thus $r_{\alpha} \sigma^{\alpha}(p) \in P$ for all $\alpha \in A$.

Therefore $r_{\alpha} \sigma^{\alpha}(P) \subseteq P$ for any $\alpha \in A$, and hence $\sigma^{\alpha}(P) \subseteq P$, because $r_{\alpha} \notin P$. Since $\sigma_{1}, \ldots, \sigma_{s}$ are independent relative to $P$, this is only possible for $\alpha=0$. Thus $A=\{0\}$, and $x=r_{0}$. Now $x$ is an element of $J \cap R$ that does not lie in $P$.

COROllary 6.2. Let $R$ be a commutative ring, let $\sigma_{1}, \ldots, \sigma_{s}$ be commuting automorphisms of $R$, and set $T=R\left[\theta_{1}^{ \pm 1}, \ldots, \theta_{s}^{ \pm 1}\right]$. Let $M$ be a maximal ideal of $R$. If $\sigma_{1}, \ldots, \sigma_{s}$ are independent relative to $M$, then $M T$ is a maximal right ideal of $T$.

Lemma 6.1 and Corollary 6.2 will be used in the context of a skew-Laurent extension $T=R\left[\theta_{1}^{ \pm 1}, \ldots, \theta_{u}^{ \pm 1}\right]$ where $R$ is a commutative noetherian ring and $\sigma_{1}, \ldots, \sigma_{u}$ are commuting automorphisms of $R$. Given a prime ideal $P$ in $R$, we may renumber the $\sigma_{i}$ so that $\sigma_{1}, \ldots, \sigma_{s}$ are independent relative to $P$, where $s=\operatorname{aut} . \operatorname{codim} .(P)$. If $S=$ $R\left[\theta_{1}^{ \pm 1}, \ldots, \theta_{s}^{ \pm 1}\right]$, then the $S$-module $S / P S$ will be made into a right module over a skew-Laurent ring

$$
U=S\left[\boldsymbol{\theta}_{s+1}^{ \pm n(s+1)}, \ldots, \boldsymbol{\theta}_{u}^{ \pm n(u)}\right],
$$


where the nonzero integers $n(j)$ (for $j=s+1, \ldots, u$ ) arise from the relations expressing the dependence of the automorphisms $\sigma_{1}, \ldots, \sigma_{s}, \sigma_{j}$ relative to $P$. To effect this procedure, we require a means of making $S$-modules into $U$-modules, and then we need to study the relations between the Krull dimensions of $U$-modules and $T$-modules.

LEMMA 6.3. Let $S$ be a ring, let $\sigma_{1}, \ldots, \sigma_{u}$ be commuting automorphisms of $S$, and set $U=R\left[\theta_{1}^{ \pm 1}, \ldots, \theta_{u}^{ \pm 1}\right]$. Let $J$ be a right ideal of $S$, let $a_{1}, \ldots, a_{u}$ be invertible elements of $S$, and assume that

$$
a_{i} \sigma_{i}^{-1}(J)=J \text { and } a_{j} \sigma_{j}^{-1}\left(a_{i}\right)-a_{i} \sigma_{i}^{-1}\left(a_{j}\right) \in J
$$

for all $i, j=1, \ldots, u$. Then $S / J$ supports a right $U$-module structure compatible with its right $S$-module structure, using a $U$-module multiplication * such that

$$
(s+J) * \theta_{l}=a_{i} \sigma_{i}^{-1}(s)+J
$$

for all $s \in S$ and all $i=1, \ldots, u$.

Proof. For a given index $i$, the rule $s \mapsto a_{i} \sigma_{l}^{-1}(s)$ defines an abelian group automorphism of $S$. Since $a_{i} \sigma_{i}^{-1}(J)=J$, this map induces an abelian group automorphism of $S / J$, which we denote $(-) * \theta_{i}$. For $r, s \in S$, we check that

$$
\begin{aligned}
{\left[(r+J) * \theta_{i}\right] s } & =\left[a_{i} \sigma_{i}^{-1}(r)+J\right] s=a_{i} \sigma_{i}^{-1}(r) s+J \\
& =a_{i} \sigma_{i}^{-1}\left(r \sigma_{i}(s)\right)+J=\left[(r+J) \sigma_{i}(s)\right] * \theta_{i} .
\end{aligned}
$$

For $s \in S$ and $i, j=1, \ldots, u$, we check that

$$
\left[(s+J) * \theta_{i}\right] * \theta_{J}=\left[a_{i} \sigma_{l}^{-1}(s)+J\right] * \theta_{j}=a_{j} \sigma_{j}^{-1}\left(a_{i}\right) \sigma_{J}^{-1} \sigma_{l}^{-1}(s)+J,
$$

and similarly $\left[(s+J) * \theta_{j}\right] * \theta_{l}=a_{i}{\sigma_{i}^{-1}}^{-1}\left(a_{j}\right) \sigma_{i}^{-1} \sigma_{j}^{-1}(s)+J$. Hence,

$$
\begin{aligned}
& {\left[(s+J) * \theta_{i}\right] * \theta_{j}-\left[(s+J) * \theta_{j}\right] * \theta_{i}} \\
& \quad=\left(a_{j} \sigma_{J}^{-1}\left(a_{i}\right)-a_{i} \sigma_{i}^{-1}\left(a_{j}\right)\right) \sigma_{j}^{-1} \sigma_{i}^{-1}(s)+J=0,
\end{aligned}
$$

because $a_{j} \sigma_{J}^{-1}\left(a_{i}\right)-a_{i} \sigma_{i}^{-1}\left(a_{j}\right) \in J$. Therefore $S / J$ does indeed become a right $U$-module in the manner described.

LEMMA 6.4. Let $S$ be a right noetherian ring, let $\sigma_{1}, \ldots, \sigma_{u}$ be commuting automorphisms of $S$, and set $T=S\left[\theta_{1}^{ \pm 1}, \ldots, \theta_{u}^{ \pm 1}\right]$. Let $n(1), \ldots, n(u)$ be nonzero integers, and set

$$
U=S\left[\theta_{1}^{ \pm n(1)}, \ldots, \theta_{u}^{ \pm n(u)}\right]
$$


If $J$ is any right ideal of $S$, then

$$
\mathrm{K} \cdot \operatorname{dim}_{{ }_{T}}(T / J T)=\mathrm{K} \cdot \operatorname{dim} \cdot_{U}(T / J T)=\mathrm{K} \cdot \operatorname{dim} ._{U}(U / J U) .
$$

Proof. Without loss of generality, we may assume that each $n(i)>0$. As a right or a left $U$-module,

$$
T=\bigoplus_{j(1)=0}^{n(1)-1} \cdots \bigoplus_{j(u)=0}^{n(u)-1} U \theta_{1}^{j(1)} \theta_{2}^{j(2)} \cdots \theta_{u}^{j(u)}
$$

and each of the monomials $\theta_{1}^{J^{(1)}} \theta_{2}^{{ }^{(2)}} \cdots \theta_{u}^{{ }^{(u)}}$ normalizes $U$. Thus $T$ is a finite normalizing extension of $U$, and hence, by [8, Théorème 5.3],

$$
\mathrm{K} \cdot \operatorname{dim}_{{ }_{T}}(T / J T)=\mathrm{K} \cdot \operatorname{dim}_{{ }_{U}}(T / J T) .
$$

As a right $U$-module,

$$
T / J T \cong \bigoplus_{j(1)=0}^{n(1)-1} \cdots \bigoplus_{j(u)=0}^{n(u)-1}\left(U \theta_{1}^{J(1)} \theta_{2}^{j(2)} \cdots \theta_{u}^{j(u)} / J U \theta_{1}^{J(1)} \theta_{2}^{j(2)} \cdots \theta_{u}^{j(u)}\right),
$$

and the lattice of submodules of each of these summands is isomorphic to the lattice of submodules of $U / J U$. Therefore K.dim. $U(T / J T)=$ K.dim. ${ }_{U}(U / J U)$.

Proposition 6.5. Let $R$ be a commutative noetherian ring, let $\sigma_{1}, \ldots, \sigma_{u}$ be commuting automorphisms of $R$, and set $T=R\left[\theta_{1}^{ \pm 1}, \ldots, \theta_{u}^{ \pm 1}\right]$. If $P$ is $a$ prime ideal of $R$ such that $R / P$ has finite Krull dimension, then

$$
\text { K.dim. }(T / P T) \geq \text { aut.dim. }(P),
$$

while if $P$ is a maximal ideal of $R$, then

$$
\text { K.dim. }(T / P T)=\text { aut.dim. }(P) \text {. }
$$

Proof. Set $s=$ aut.codim. $(P)$, and renumber the $\sigma_{l}$ so that $\sigma_{1}, \ldots, \sigma_{s}$ are independent relative to $P$. Set $S=R\left[\theta_{1}^{ \pm 1}, \ldots, \theta_{s}^{ \pm 1}\right]$.

For each $j=s+1, \ldots, u$, the automorphisms $\sigma_{1}, \ldots, \sigma_{s}, \sigma_{j}$ are dependent relative to $P$. Hence, there exist integers $m(j, 1), \ldots, m(j, s), n(j)$, with $n(j) \neq 0$, such that

$$
\boldsymbol{\sigma}_{1}^{m(j, 1)} \boldsymbol{\sigma}_{2}^{m(J, 2)} \cdots \sigma_{s}^{m(j, s)} \boldsymbol{\sigma}_{j}^{n(J)}(P) \subseteq P .
$$

Since the ring

$$
R_{j}=R / \boldsymbol{\sigma}_{1}^{m(j, 1)} \boldsymbol{\sigma}_{2}^{m(J, 2)} \cdots \boldsymbol{\sigma}_{s}^{m(j, s)} \boldsymbol{\sigma}_{j}^{n(j)}(P)
$$


is a domain isomorphic to $R / P$, it has the same Krull dimension as $R / P$, and this Krull dimension is finite. Hence, $R / P$ cannot be a proper homomorphic image of $R_{j}$, and so

$$
\boldsymbol{\sigma}_{1}^{m(J, 1)} \boldsymbol{\sigma}_{2}^{m(j, 2)} \cdots \boldsymbol{\sigma}_{s}^{m(J, s)} \boldsymbol{\sigma}_{J}^{n(J)}(P)=P .
$$

For $j=s+1, \ldots, u$, set

$$
a_{j}=\theta_{1}^{m(J, 1)} \theta_{2}^{m(J, 2)} \cdots \theta_{s}^{m(J, s)},
$$

which is an invertible element of $S$, and observe that

$$
\begin{aligned}
a_{j} \boldsymbol{\sigma}_{j}^{n(J)}(P S) & =\sigma_{1}^{m(J, 1)} \boldsymbol{\sigma}_{2}^{m(J, 2)} \cdots \sigma_{s}^{m(J, s)} \boldsymbol{\sigma}_{J}^{n(J)}(P) \boldsymbol{\theta}_{1}^{m(j, 1)} \boldsymbol{\theta}_{2}^{m(J, 2)} \cdots \boldsymbol{\theta}_{s}^{m(J, s)} S \\
& =P S .
\end{aligned}
$$

In addition, for $j, k=s+1, \ldots, u$ we have

$$
\begin{aligned}
a_{j} \sigma_{j}^{n(J)}\left(a_{k}\right) & =\theta_{1}^{m(J, 1)} \theta_{2}^{m(J, 2)} \cdots \theta_{s}^{m(j, s)} \theta_{1}^{m(k, 1)} \theta_{2}^{m(k, 2)} \cdots \theta_{s}^{m(k, s)} \\
& =a_{k} \sigma_{k}^{n(k)}\left(a_{J}\right),
\end{aligned}
$$

and so

$$
a_{j} \sigma_{j}^{n(J)}\left(a_{k}\right)-a_{k} \sigma_{k}^{n(k)}\left(a_{J}\right) \in P S .
$$

We now set $U=S\left[\theta_{s+1}^{ \pm n(s+1)}, \ldots, \theta_{u}^{ \pm n(u)}\right]$, viewed as a skew-Laurent extension of $S$ with respect to the automorphisms $\sigma_{s+1}^{-n(s+1)}, \ldots, \sigma_{u}^{-n(u)}$. Using Lemma 6.3, the module $S / P S$ can be made into a right $U$-module in a manner compatible with its right $S$-module structure. By Proposition 5.2

$\mathrm{K} \cdot \operatorname{dim} \cdot(U / P U) \geq \mathrm{K} \cdot \operatorname{dim}_{\cdot}(S / P S)+(u-s) \geq u-s$.

Then, applying Lemma 6.4, we conclude that

$\mathrm{K} \cdot \operatorname{dim} .(T / P T)=\mathrm{K} \cdot \operatorname{dim} .(U / P U) \geq u-s=\operatorname{aut} \cdot \operatorname{dim} .(P)$.

Finally, assume that $P$ is a maximal ideal of $R$. In this case, Corollary 6.2 shows that $P S$ is a maximal right ideal of $S$, and so $S / P S$ is a simple right $S$-module. Hence,

$\mathrm{K} \cdot \operatorname{dim} .(T / P T) \leq \mathrm{K} \cdot \operatorname{dim} .(S / P S)+(u-s)=u-s$, and therefore K.dim. $(T / P T)=u-s$.

VII. Krull dimension formulas. We are now in a position to derive a formula for the Krull dimension of a skew-Laurent extension $T=$ $R\left[\theta_{1}^{ \pm 1}, \ldots, \theta_{u}^{ \pm 1}\right]$ over a commutative noetherian ring $R$ of finite Krull dimension, equipped with $u$ commuting automorphisms. If $Q$ is a prime 
ideal of $R$, then $R / Q$ is a compressible $R$-module. Hence, by Corollary 1.3 , each of the modules $(R / Q) \otimes_{R} R\left[\theta_{1}^{ \pm 1}, \ldots, \theta_{k}^{ \pm 1}\right]$ (for $k=1, \ldots, u$ ) is compressible, and thus critical. Consequently, $R / Q$ is a $u$-clean $R$-module. Since $T / Q T$ is a critical $T$-module,

$$
\text { K.dim. }(T / Q T)>\mathrm{K} \cdot \operatorname{dim} .(T / P T)
$$

for any prime ideal $P$ of $R$ that properly contains $Q$. Applying this observation to descending chains of prime ideals of $R$, we obtain

$$
\text { K.dim. }(T) \geq \mathrm{K} \cdot \operatorname{dim} .(T / P T)+\mathrm{ht}(P)
$$

for any prime ideal $P$ of $R$. As a result, our work in the previous section provides us with lower bounds for the Krull dimension of $T$, namely

$$
\text { K.dim. }(T) \geq \operatorname{aut.dim} .(P)+\mathrm{ht}(P)
$$

for all prime ideals $P$ of $R$. In this section, we show that K.dim.(T) is actually the maximum of these lower bounds.

Proposition 7.1. Let $R$ be a commutative noetherian ring, let $\sigma_{1}, \ldots, \sigma_{u}$ be commuting automorphisms of $R$, and set $T=R\left[\theta_{1}^{ \pm 1}, \ldots, \theta_{u}^{ \pm 1}\right]$. Let $P$ be $a$ non-maximal prime ideal of $R$ such that $\mathrm{K} \cdot \operatorname{dim} .(R / P)$ is finite, and set

$$
l=\max \{\mathrm{K} \cdot \operatorname{dim} .(T / Q T) \mid Q \in \operatorname{Spec}(R) \text { and } Q>P\} .
$$

If $\mathrm{K} \cdot \operatorname{dim} .(T / P T)>$ aut.dim. $(P)$, then $\mathrm{K} \cdot \operatorname{dim} .(T / P T)=l+1$.

Proof. Set $m=$ K.dim. $(T / P T)$ and $s=\operatorname{aut.codim} .(P)$. Then $m>$ $u-s$ by hypothesis, and $m \geq l+1$ because $T / P T$ is critical. Renumber the $\sigma_{i}$ so that $\sigma_{1}, \ldots, \sigma_{s}$ are independent relative to $P$, and set $S=$ $R\left[\theta_{1}^{ \pm 1}, \ldots, \theta_{s}^{ \pm 1}\right]$. By Lemma 6.1 , if $J$ is any right ideal of $S$ such that $J>P S$, then $J \cap R>P$.

Now the module $B=S / P S$ is a compressible noetherian right $S$ module, by Corollary 1.3. Also, by the same result, $B$ is $(u-s)$-clean. Since $B \otimes_{S} T \cong T / P T$, we have

$$
\text { K.dim. }\left(B \otimes_{S} T\right)=m>u-s .
$$

Hence, by Proposition 5.5, there exists a $(u-s)$-clean minor subfactor $A$ of $B$ such that

$$
\text { K.dim. }\left(A \otimes_{S} T\right)=m-1 .
$$

Let $I>J>P S$ be right ideals of $S$ such that $I / J=A$. Then $J \cap R$ $>P$, and so $(J \cap R) T>P T$, whence

$$
\text { K.dim. }(T /(J \cap R) T)<\mathrm{K} \cdot \operatorname{dim} .(T / P T)=m,
$$


because $T / P T$ is critical. However, $T /(J \cap R) T$ has a subfactor isomorphic to $I T / J T$, which is isomorphic to $A \otimes_{S} T$, and hence

$$
\mathrm{K} \cdot \operatorname{dim} .(T /(J \cap R) T) \geq \mathrm{K} \cdot \operatorname{dim} .\left(A \otimes_{S} T\right)=m-1 .
$$

Thus, K.dim. $(T /(J \cap R) T)=m-1$.

There is a chain

$$
P<J \cap R=J_{0}<J_{1}<\cdots<J_{n}=R
$$

of ideals of $R$ such that each $J_{i} / J_{i-1} \cong R / Q_{i}$ for some prime ideal $Q_{i}>P$ in $R$. Thus

$$
\mathrm{K} \cdot \operatorname{dim} .\left(T / Q_{l} T\right) \leq l
$$

for each $i$. Now there is a chain of right ideals

$$
(J \cap R) T=J_{0} T<J_{1} T<\cdots<J_{n} T=T
$$

in $T$ such that each $J_{i} T / J_{l-1} T \cong T / Q_{l} T$, and so

$$
\mathrm{K} \cdot \operatorname{dim} .\left(T / Q_{l} T\right)=m-1
$$

for some $i$, because $\mathrm{K} \cdot \operatorname{dim} .(T /(J \cap R) T)=m-1$. Hence, $m-1 \leq l$, and therefore $m=l+1$.

THEOREM 7.2. Let $R$ be a commutative noetherian ring, let $\sigma_{1}, \ldots, \sigma_{u}$ be commuting automorphisms of $R$, and set $T=R\left[\theta_{1}^{ \pm 1}, \ldots, \theta_{u}^{ \pm 1}\right]$. For each prime ideal $P$ of $R$, set

$$
m_{P}=\sup \left\{\mathrm{ht}(Q / P)+\text { aut.dim. }(Q) \mid Q \in \mathfrak{N}_{P}\right\},
$$

where $\Re_{P}$ is the set of those prime ideals of $R$ that contain $P$. If $R / P$ has finite Krull dimension, then K.dim. $(T / P T)=m_{P}$.

Proof. The proof is by induction on K.dim. $(R / P)$. If K.dim. $(R / P)$ $=0$, then $P$ is a maximal ideal of $R$, and the result follows immediately from Proposition 6.5.

Now assume that K.dim. $(R / P)>0$, and that the result holds for all prime ideals properly containing $P$.

Consider any $Q \in \Re_{P}$. If $Q=P$, then by Proposition 6.5,

K.dim. $(T / P T) \geq$ aut.dim. $(Q)=\operatorname{ht}(Q / P)+\operatorname{aut.dim} .(Q)$.

If $Q>P$, then there exists a prime ideal $Q^{\prime}$ of $R$ such that $Q \geq Q^{\prime}>P$ and $\operatorname{ht}\left(Q / Q^{\prime}\right)=\operatorname{ht}(Q / P)-1$. Then,

K.dim. $\left(T / Q^{\prime} T\right)=m_{Q^{\prime}} \geq \mathrm{ht}\left(Q / Q^{\prime}\right)+\operatorname{aut} \cdot \operatorname{dim} .(Q)$

$$
=\operatorname{ht}(Q / P)+\text { aut.dim. }(Q)-1
$$


by the induction hypothesis, and so

K.dim. $(T / P T) \geq \mathrm{K} \cdot \operatorname{dim} .\left(T / Q^{\prime} T\right)+1 \geq \mathrm{ht}(Q / P)+\operatorname{aut} \cdot \operatorname{dim} .(Q)$, since $T / P T$ is a critical module. Thus, $\mathrm{K} \cdot \operatorname{dim} .(T / P T) \geq m_{P}$.

If $\mathrm{K} . \operatorname{dim} .(T / P T) \leq$ aut.dim. $(P)$, then K.dim. $(T / P T) \leq m_{P}$, and the result holds in this case. Thus we may assume that

$$
\text { K.dim. }(T / P T)>\text { aut.dim. }(P) \text {. }
$$

Then, by Proposition 7.1, there exists a prime ideal $Q>P$ such that

$$
\mathrm{K} \cdot \operatorname{dim} \cdot(T / P T)=\mathrm{K} \cdot \operatorname{dim} \cdot(T / Q T)+1 .
$$

Applying the induction hypothesis, we obtain

$$
\mathrm{K} \cdot \operatorname{dim} .(T / P T)=m_{Q}+1 \leq m_{P},
$$

and therefore the result holds in this case also.

The main result now follows easily from Theorem 7.2.

THEOREM 7.3. Let $R$ be a commutative noetherian ring with finite Krull dimension, let $\sigma_{1}, \ldots, \sigma_{u}$ be commuting automorphisms of $R$, and set $T=$ $R\left[\theta_{1}^{ \pm 1}, \ldots, \theta_{u}^{ \pm 1}\right]$. Then

$$
\text { K.dim. }(T)=\max \{\operatorname{ht}(Q)+\text { aut.dim. }(Q) \mid Q \in \operatorname{Spec}(R)\} .
$$

Proof. Let $P_{1}, \ldots, P_{k}$ be the minimal prime ideals of $R$. Since $R$ has a finite chain of ideals from 0 to $R$ such that the successive subfactors are homomorphic images of the modules $R / P_{i}$, we see that

$$
\mathrm{K} \cdot \operatorname{dim} .(T)=\max \left\{\mathrm{K} \cdot \operatorname{dim} .\left(T / P_{l} T\right) \mid i=1, \ldots, k\right\} .
$$

Now for each prime ideal $Q$ of $R$,

$$
\operatorname{ht}(Q)=\max \left\{\operatorname{ht}\left(Q / P_{i}\right) \mid i=1, \ldots, k \text { and } P_{i} \leq Q\right\},
$$

and hence the result follows from Theorem 7.2.

The analogue of Theorem 7.3 for differential operator rings in characteristic zero, [4, Corollary 4.4], has a useful simplification in the case that the coefficient ring is a finitely generated algebra over a field of characteristic zero. Namely, in that case the maximum value in the formula for the Krull dimension of the differential operator ring occurs at a maximal ideal of the coefficient ring [4, Theorem 6.8]. In the skewLaurent case, no such simplification occurs, as the following example shows. 
Let $x$ be an indeterminate, and let $R$ be the polynomial ring $\mathbb{C}[x]$. Let $\sigma_{1}$ and $\sigma_{2}$ be the $\mathbf{C}$-algebra automorphisms of $R$ such that $\sigma_{1}(x)=x+1$ and $\sigma_{2}(x)=x+\pi$, and set $T=R\left[\theta_{1}^{ \pm 1}, \theta_{2}^{ \pm 1}\right]$. Since 1 and $\pi$ are $\mathbb{Z}$-linearly independent, we infer that $\sigma_{1}$ and $\sigma_{2}$ are independent relative to any maximal ideal of $R$. Hence,

$$
\operatorname{ht}(M)+\text { aut.dim. }(M)=1
$$

for all maximal ideals $M$ of $R$. However, $h t(0)+$ aut.dim. $(0)=2$, and so K.dim. $(T)=2$.

\section{REFERENCES}

[1] G. Bonnefond, Sur la dimension de Krull de A[X, $X^{-1}$; $\left.\sigma\right]$, C. R. Acad. Sci. Paris, Sér. A, 286 (1978), 759-762.

[2] K. R. Goodearl, T. J. Hodges, and T. H. Lenagan, Krull and global dimensions of Weyl algebras over division rings, J. Algebra (to appear).

[3] K. R. Goodearl and T. H. Lenagan, Krull dimension of differential operator rings. III-Noncommutative coefficients, Trans. Amer. Math. Soc., 275 (1983), 833-859.

[4] Krull dimension of differential operator rings. IV-Multiple derivations, Proc. London Math. Soc. (3) 47 (1983), 306-336.

[5] R. Gordon and J. C. Robson, Krull dimension, Memoirs Amer. Math. Soc., No. 133 (1973).

[6] T. J. Hodges, The Krull dimension of skew-Laurent extensions of commutative noetherian rings, Communic. in Algebra (to appear).

[7] T. Hodges and J. C. McConnell, On Ore and skew-Laurent extensions of noetherian rings, J. Algebra, 73 (1981), 56-64.

[8] B. Lemonnier, Dimension de Krull et codeviation. Application au théorème d'Eakin, Comm. Algebra, 6 (1978), 1647-1665.

[9] R. Rentschler and P. Gabriel, Sur la dimension des anneaux et ensembles ordonnés, C. R. Acad. Sci. Paris, Sér. A, 265 (1967), 712-715.

[10] P. F. Smith, Corrigendum to 'On the dimension of group rings', Proc. London Math. Soc., (3) 27 (1973), 766-768.

Received September 24, 1982. The research of the first author was partially supported by a grant from the National Science Foundation.

UNIVERSITY OF UTAH

SALT LAKE CITY, UT 84112

AND

UNIVERSITY OF EDINBURGH

J.C.M.B., KINGS BUILDINGS

MAYFIELD ROAD, EDINBURGH

EH9 3JZ SCOTLAND 



\section{PACIFIC JOURNAL OF MATHEMATICS \\ EDITORS}

DONALD BABBITt (Managing Editor)

University of California

Los Angeles, CA 90024

J. Dugundu

University of Southern California

Los Angeles, CA 90089-1113

R. FINN

Stanford University

Stanford, CA 94305

HERMANN FLASCHKA

University of Arizona

Tucson, AZ 85721
C. C. MOORE

University of California

Berkeley, CA 94720

ARTHur Ogus

University of California

Berkeley, CA 94720

Hugo RossI

University of Utah

Salt Lake City, UT 84112

H. SAMELSON

Stanford University

Stanford, CA 94305

ASSOCIATE EDITORS

R. ARENS

E. F. BECKENBACH

B. H. NeumanN (1906-1982)

F. WOLF

K. YoshidA

\section{SUPPORTING INSTITUTIONS}

UNIVERSITY OF ARIZONA

UNIVERSITY OF BRITISH COLUMBIA

UNIVERSITY OF OREGON

CALIFORNIA INSTITUTE OF TECHNOLOGY

UNIVERSITY OF CALIFORNIA

MONTANA STATE UNIVERSITY

UNIVERSITY OF SOUTHERN CALIFORNIA

UNIVERSITY OF NEVADA, RENO

STANFORD UNIVERSITY

UNIVERSITY OF HAWAII

NEW MEXICO STATE UNIVERSITY

UNIVERSITY OF TOKYO

UNIVERSITY OF UTAH

OREGON STATE UNIVERSITY

WASHINGTON STATE UNIVERSITY

UNIVERSITY OF WASHINGTON 


\section{Pacific Journal of Mathematics}

Vol. 114, No. $1 \quad$ May, 1984

David Marion Arnold and Charles Irvin Vinsonhaler, Typesets and cotypesets of rank-2 torsion free abelian groups $\ldots \ldots \ldots \ldots \ldots \ldots \ldots 1$

Duncan Alan Buell and Richard Howard Hudson, Solutions of certain

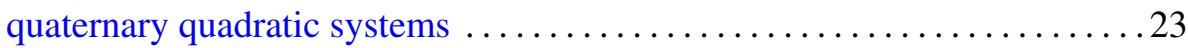

Hans Delfs and Manfred Knebusch, Separation, retractions and homotopy

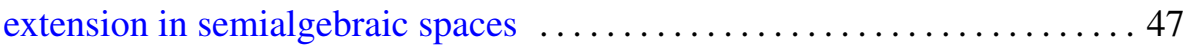

I. Erdélyi and Sheng-Wang Wang, A spectral duality theorem for closed

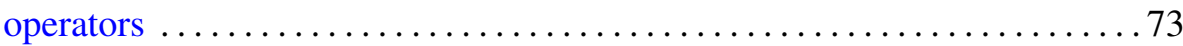

Theodore William Gamelin, Weak compactness of representing measures for $R(K)$

Kenneth R. Goodearl and T. H. Lenagan, Krull dimension of

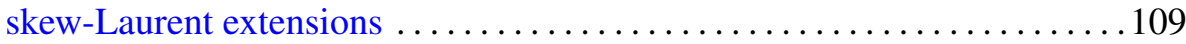

Daniel Joseph Gross, Compact quotients by $\mathbf{C}^{*}$-actions . . . . . . . . . . . 149

Goo Ishikawa, Satoshi Koike and Masahiro Shiota, Critical value sets of

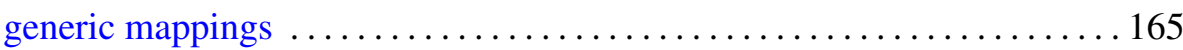

Hong Oh Kim, Derivatives of Blaschke products $\ldots \ldots \ldots \ldots \ldots \ldots \ldots \ldots \ldots$

Erhard Luft and Denis Karmen Sjerve, 3-manifolds with subgroups

$Z \oplus Z \oplus Z$ in their fundamental groups $\ldots \ldots \ldots \ldots \ldots \ldots \ldots \ldots \ldots \ldots \ldots \ldots$

George Clifford Nelson, Boolean powers, recursive models, and the Horn

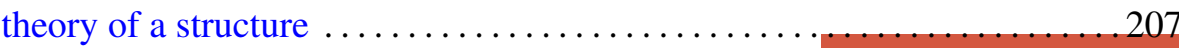

W. J. Phillips, Flow under a function and discrete decomposition of properly infinite $W^{*}$-algebras

Teodor C. Przymusiński, A solution to a problem of E. Michael ... 235

Bruce Harvey Wagner, Derivations of quasitriangular algebras 243 